\title{
Synthesis of [(1,2,3-Triazol-1-yl)methyl]boronic Acids Through Click Chemistry: Easy Access to a Potential Scaffold for Protease Inhibitors
}

\author{
Chiara Romagnoli, ${ }^{[a]}$ Emilia Caselli, ${ }^{[a]}$ and Fabio Prati*[a]
}

\author{
Keywords: Synthetic methods / Boron / Cycloaddition / Click chemistry / Bioisosters / Medicinal chemistry / Enzyme \\ inhibitors
}

Stereoselective synthesis of previously unreported $[(1,2,3-$ triazol-1-yl)methyl]boronic acids has been achieved from azidomethylboronates by copper-catalyzed azide-alkyne cycloaddition reaction. The proximity of the cycloaddition reaction center to the boronic group is not detrimental to the stability of the $s p^{3} \mathrm{C}-\mathrm{B}$ bond or to the stereoisomeric composition, which further expands the field of application of click chemistry to new boronate substrates and offers a new potential scaffold for protease inhibitors.

\section{Introduction}

$\alpha$-Amidomethylboronic acid is a recurring core-structure in biologically active and important boron-containing compounds. ${ }^{[1]}$ After the approval of Velcade ${ }^{\circledR}$ (Figure 1) and clarification on the safety issues relating to boron-containing compounds, the use of this element in pharmaceutical research has become an attractive "hot" topic. As a result, several boron derivatives are currently in preclinical and clinical stage development. ${ }^{[2]}$ Among other compounds, boronic acids exhibit excellent properties as competitive and reversible protease inhibitors. Owing to their unique structural features, they act as transition state analogs: boron, with its open shell, interacts with nucleophilic active residues and in doing so converts from a neutral trigonal structure into an anionic tetrahedral adduct, which mimics the high-energy intermediate in the amide hydrolysis process.

31 The boron moiety acts as the "warhead" blocking the catalytic site, whereas the $\alpha$-amido group enhances molecular recognition by mimicking natural substrates. ${ }^{[3]}$

The $\alpha$-amidomethylboronate unit is the basic structure of peptidoboronic acids, a class of peptidomimetics largely explored to target different clinically relevant proteases. For example, anticancer compound Velcade ${ }^{\circledR}(\mathbf{A})$ is a dipeptidylboronic acid (Phe-boroLeu) that acts as a proteasome inhibitor, whereas derivatives of the type Val-boroPro or ProboroAla have been investigated as dipeptidyl peptidase- 4 inhibitors for the treatment of diabetes. ${ }^{[4]}$ The same skeleton is also part of simpler acylamidomethylboronic acids, which have been reported to be subtilisin and $\alpha$-chymotripsin in-

[a] Dipartimento di Scienze della Vita, Università degli Studi di Modena e Reggio-Emilia,

Via Campi 183, 41125 Modena (MO)

E-mail: fabio.prati@unimore.it http://www.unimore.it

Supporting information for this article is available on the WWW under http://dx.doi.org/10.1002/ejoc.201403408.

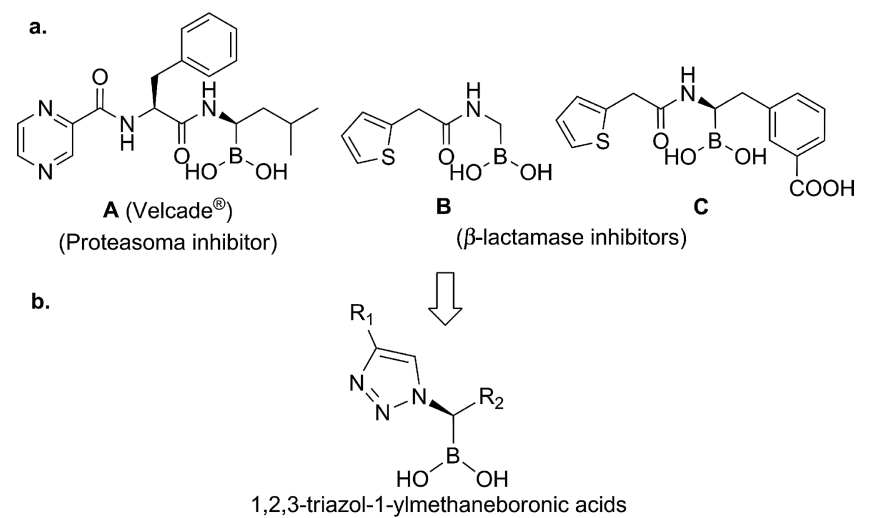

Figure 1. $\boldsymbol{a}$. Examples of $\alpha$-amidomethylboronic acids found in biologically active compounds. $\boldsymbol{b}$. Bioisosteric amide replacement with 1,4-disubstituted 1,2,3-triazoles.

hibitors and used as fluorescent carbohydrate sensors. ${ }^{[5]}$ In accordance with these developments, we investigated acylamidomethylboronic acids $\mathbf{B}$ and $\mathbf{C}$ (Figure 1, a) as potent and selective $\beta$-lactamase inhibitors. ${ }^{[6]}$

During our investigation, we were intrigued by the effect of $\alpha$-amido group replacement with 1,4-disubstituted 1,2,3triazole, which is a known non-classical amide bioisoster (Figure 1, b). These two groups share several chemical properties such as planarity, size, dipole moment and hydrogen bond forming capabilities. However, they also have important differences: triazole hopping can restrict conformational flexibility and improve hydrolysis and oxidation stability. ${ }^{[7]}$ Furthermore, 1,4-disubstituted 1,2,3-triazoles are easily accessible through copper-catalyzed azidealkyne cycloaddition ( $\mathrm{CuAAC})$ reaction, which is a preferred protocol because it proceeds under mild conditions with inexpensive reagents, and with high versatility, high efficiency, and straightforward product isolation. ${ }^{[8]}$ For these reasons, click chemistry is increasing in popularity in 
drug discovery and the use of triazole units as amide surrogates is becoming routine in structure activity relationship studies. ${ }^{[9]}$

Surprisingly, this strategy has never been applied to $\alpha$ amidomethylboronic acids, and [(1,2,3-triazol-1-yl)methyl]boronic acids are an unexplored scaffold. Investigation in this field has probably been discouraged by the known ability of copper to insert into the $\mathrm{C}-\mathrm{B}$ bond. Actually, this activation is exploited in several useful cross-coupling reactions, such as in a copper-variant of the Suzuki-Miyaura reaction and in the Chan-Lam $\mathrm{C}-\mathrm{N}$ and $\mathrm{C}-\mathrm{O}$ coupling reaction with boronates. ${ }^{[10]}$ However, these methods can lead to degradation-promoting protodeboronation. To date, some efforts on click reactions applied to boron derivatives have been done, but these have been restricted to arylboronates, probably owing to their greater availability and stability. Moreover, in these cases the alkyne and azide involved in the CuAAC reaction are remote with respect to the boronic moiety and the few examples reported in the literature proceed with some difficulties $\square((<=$ Author: do you agree with the change?)) $\mathbf{\square}$. Some unique approaches have been tried, such as fluoride addition to stabilize the $\mathrm{C}-\mathrm{B}$ bond or inversion of the step sequence to insert the boron atom after the $\mathrm{CuAAC}$ reaction. ${ }^{[11]}$ The literature reports a single case in which the boronic group was directly linked to the alkyne and produced a 1,2,3-triazole-4boronate. ${ }^{[12]}$

The first milestone of our project was to assess the feasi-

91 bility of synthesis of [(1,2,3-triazol-1-yl)methyl]boronic acids.

\section{Results and Discussion}

Initial experiments focused on the synthesis of the simplest boronic ester, which corresponding to triazolyl analogs of acyl-boroGly (Scheme 1).

By starting from pinanediyl (chloromethyl)boronate (1), ${ }^{[13]}$ substitution with sodium azide was catalyzed by tetrabutylammonium iodide, as a phase transfer agent, to yield the (azidomethyl)boronate $2(97 \%) .{ }^{[14]}$ The use of $(+)-$

101 pinanediol to esterify the boronic acid group is justified by its strong stability to hydrolysis, which allows the use of TLC as a reaction monitoring method. An investigation of $\mathrm{CuAAC}$ reaction feasibility on azido intermediate $\mathbf{2}$ was performed with ethyl propiolate as the acetylene counter106 part, which was chosen because of the general observation that $\alpha$-carbonyl groups are more reactive than alkyl- or aryl-alkynes. ${ }^{[15]}$ From the wide variety of conditions described in the literature for $\mathrm{CuAAC}$ reactions, we selected three. In two cases the copper(I) catalyst was added directly in the presence of a ligand [CuI, $N, N$-diisopropylethylamine (DIPEA), tetrahydrofuran (THF), or CuI, lutidine, $\left.\mathrm{CHCl}_{3}\right]^{\left[{ }^{[16]}\right.}$ In the third case, the catalytically active metal was generated in situ by reduction of copper sulfate $\left(\mathrm{CuSO}_{4}\right.$, sodium ascorbate, tert-butanol, $\left.\mathrm{H}_{2} \mathrm{O}\right) .{ }^{[8]}$ Each experiment was performed at room temperature for $6 \mathrm{~h}$ with a molar ratio of $2 /$ ethyl propiolate/catalyst 1:1.5:0.1. The crude product was analyzed by ${ }^{1} \mathrm{H}$ NMR spectroscopy and LC-MS, and formation of expected and previously unreported product 3a in almost complete conversion was observed in all of the three experiments, which confirms the robustness of the CuAAC reaction. Nevertheless, when the $\mathrm{CuI}$ catalyst was adopted, the NMR spectra revealed the presence of proto-deboronation by-products (5-20\%), and these were more pronounced when more basic DIPEA rather than lutidine was used as ligand. However DIPEA could be easily removed from the crude mixture under reduced pressure, whereas lutidine could not. Superior performance in terms of purity of the recovered material and absence of deboronation by-products was observed under aqueous conditions. Consequently there conditions wer $\Omega^{31}$ therefore applied to the cycloaddition reactions of 2 with several other alkynes. Among the many compounds commercially available, carbonyl, aromatic, and aliphatic alkynes were chosen. In the optimized procedure, azide $\mathbf{2}$ and an excess of alkyne ( 1.5 equiv.) were dissolved in a 1:1 mixture of tert-butanol and water, together with copper sulfate ( 0.05 equiv.), which was reduced in situ by sodium ascorbate ( 0.2 equiv.). The cyclization reactions were carried out at room temperature and monitored by TLC until no azidomethylboronate $\mathbf{2}$ remained. Complete conversion was reached in two hours with propiolic acid and ethyl propiolate (Table 1, Entries 1-2), whereas longer reaction times (up to $16 \mathrm{~h}$ ) were required for alky- and aryl-alkynes (Table 1, Entries 3-5). The expected 1,4-disubstituted triazoles were easily isolated by extraction, and any residual alkyne was removed under reduced pressure, to afford $\mathbf{3 a}-$ $3 \mathbf{e}$ in good to excellent yields (85-99\%) with high purity. The cyclic product was confirmed by the presence of a singlet signal downfield in the aromatic region in the ${ }^{1} \mathrm{H}$ NMR spectra and the expected 1,4-regioselectivity was supported by bidimensional spectroscopy [particularly the ${ }^{3} J(\mathrm{C}, \mathrm{H})$ correlation between protons on the boron-bearing carbon atom and the unsubstituted carbon of the triazole ring].

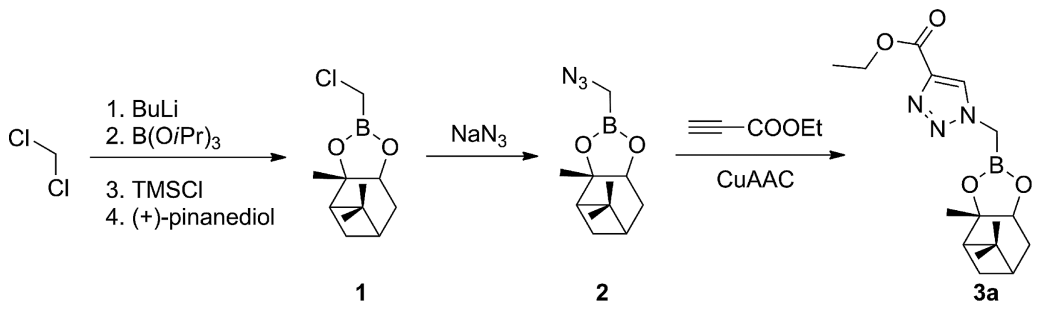

Scheme 1. Synthesis of triazolyl analog of acyl-boroGly 3a. Tested conditions for the CuAAC reaction: a) CuI, DIPEA, THF; b) CuI, lutidine, $\mathrm{CHCl}_{3} ;$ c) $\mathrm{CuSO}_{4}$, sodium ascorbate, $t \mathrm{BuOH}, \mathrm{H}_{2} \mathrm{O} ; \mathrm{TMSCl}=$ trimethylsilyl chloride. 
Final deprotection of (+)-pinanediol was accomplished by transesterification with phenylboronic acid in a biphasic system of acetonitrile/ $n$-hexane, ${ }^{[17]}$ to give desired boronic acids $\mathbf{4 a}-\mathbf{4 e}$, which were purified by crystallization from acetonitrile $(80-100 \%)$.

Table 1. Copper-catalyzed azide-alkyne cycloaddition reaction between $\alpha$-azidomethylboronate $\mathbf{2}$ and several alkynes.

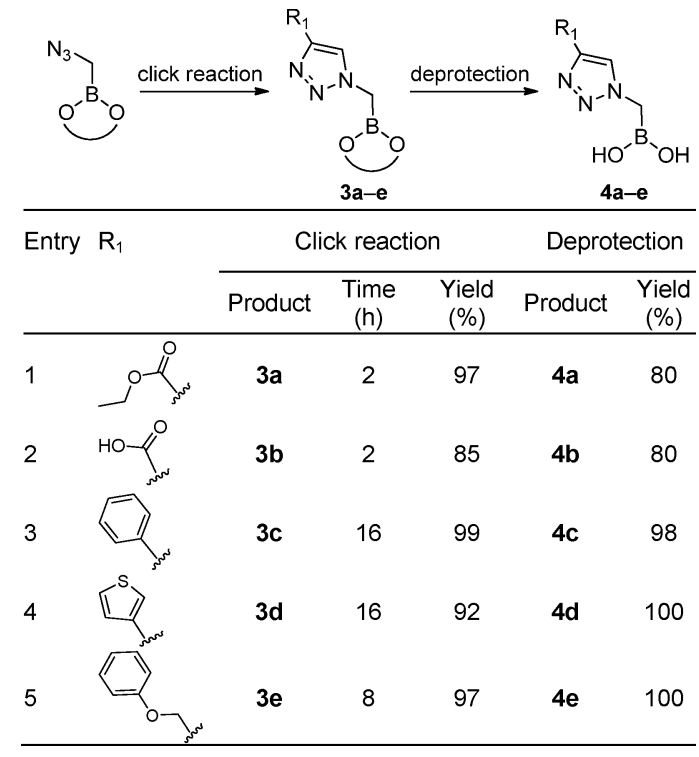

The successful synthesis of these [(1,2,3-triazol-1-yl)methyl]boronic acids prompted us to expand our project toward chiral compounds and introduce a $\mathrm{R}_{2}$-substituent (see Figure 1, b). To obtain a homochiral series with natural amino acids a stereoselective synthesis was required. At first we focused on triazolyl analogs of acyl-boroLeu that bear that is also part of $\operatorname{Velcade}^{\circledR}(\mathrm{A})$.

The configuration of the carbon in $\alpha$ position to the boron is controlled through Matteson's homologation of boronic esters, when (+)-pinanediol is used as the chiral auxiliary agent. ${ }^{[18]}$ Following this procedure, isobutylboronate 5 was treated with dichloromethyllithium generated in situ at $-100{ }^{\circ} \mathrm{C}$ for the insertion of a halogenated and asymmetrically substituted carbon on the $\mathrm{C}-\mathrm{B}$ bond. According to the literature, the use of (+)-pinanediol in $\mathbf{6}$ induced the $>98 \%$, yield 70\%). ${ }^{[19]}$ Subsequent substitution with so-

dium azide afforded azido boronate 7 (de $>98 \%$, yield $97 \%$ ). With respect to the synthesis of $\mathbf{2}$, the presence of a stereogenic center at the reactive site prevents the use of tetrabutylammonium iodide (TBAI) in favor of non-nucleo-

Table 2. Copper-catalyzed azide-alkyne cycloaddition reaction between chiral $\alpha$-azidomethylboronates and alkynes.
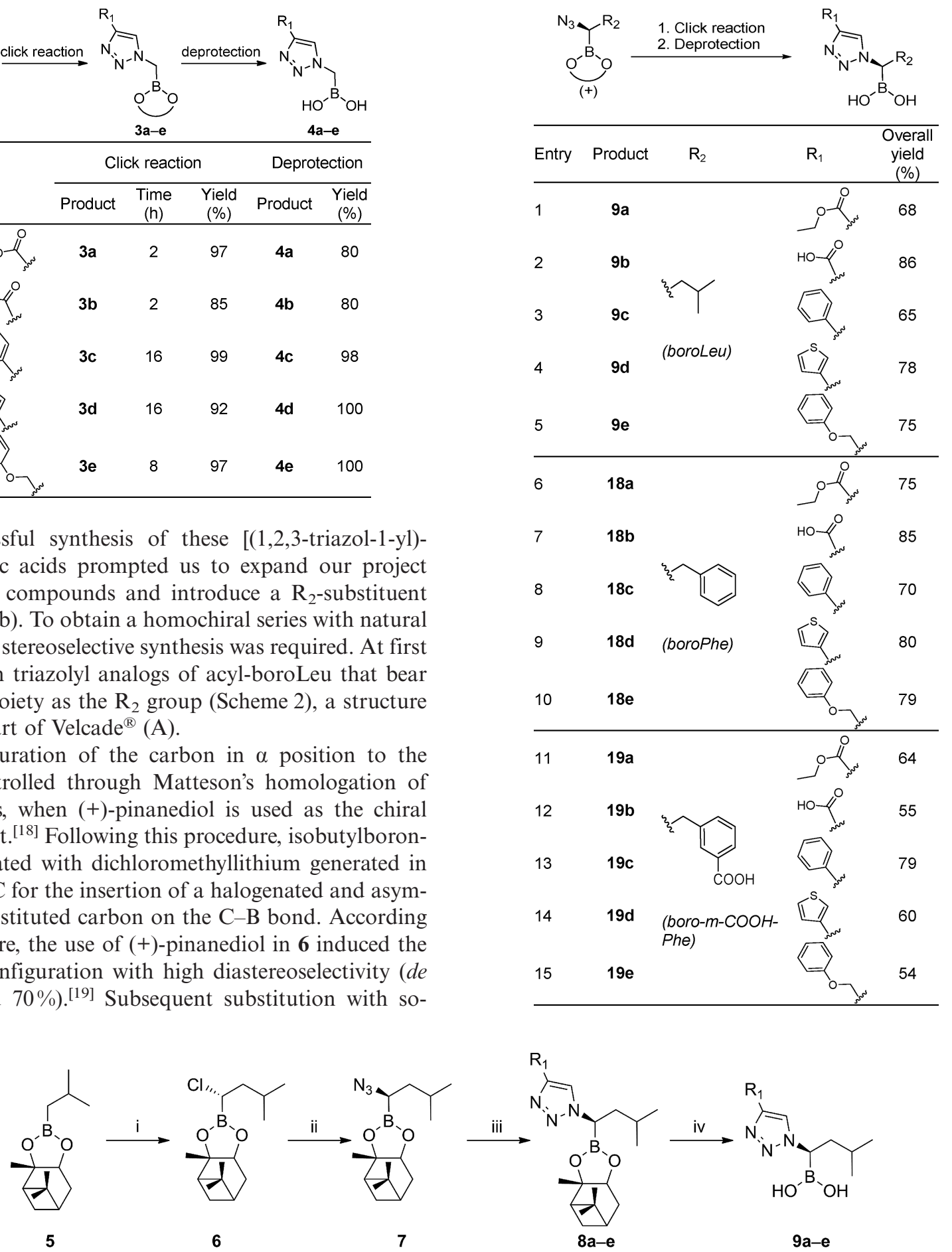

Scheme 2. Stereoselective synthesis of triazolyl analogs of acyl-boroLeu. (i) $\mathrm{LiCHCl}_{2}, \mathrm{ZnCl}_{2}, \mathrm{THF},-100{ }^{\circ} \mathrm{C} \rightarrow$ room temp.; (ii) $\mathrm{NaN} \mathrm{N}_{3}$, TBAHS, EtOAc, $\mathrm{H}_{2} \mathrm{O}$, room temp.; (iii) alkyne, $\mathrm{CuSO}_{4}$, sodium ascorbate, $t \mathrm{BuOH}, \mathrm{H}_{2} \mathrm{O}$, room temp.; (iv) phenylboronic acid, $\mathrm{HCl}$, acetonitrile, $n$-hexane, room temp. 
philic tetrabutylammonium hydrogensulfate (TBAHS) to avoid epimerization ( $30 \%$ of undesired epimer was obtained with TBAI). Click reactions to give $\mathbf{8 a}-\mathbf{8 e}$ under the same conditions described for the synthesis of $\mathbf{3 a}-\mathbf{3 e}$ performed equally well without any effect on reaction time $(2-16 \mathrm{~h}$, see the Experimental Section) or yield (81-97\%). Most importantly, no effect on the diastereoisomeric composition was observed in the NMR spectra, which was evaluated through analyses of the spectra of $\mathbf{8 a}-\mathbf{8 e}$ obtained from an epimeric mixture of 7. Final deprotection afforded enantiomerically pure triazolyl boronic acids 9a-9e (Table 2, Entries 1-5).

The same procedure was replicated for the synthesis of boroPhe analogs 18a-18e and 19a-19e (Scheme 3), which bear as the $\mathrm{R}_{2}$ group a benzyl or its meta-carboxy derivative; the latter of which is a recurring motif in $\beta$-lactamase inhibitors (Figure 1, C).

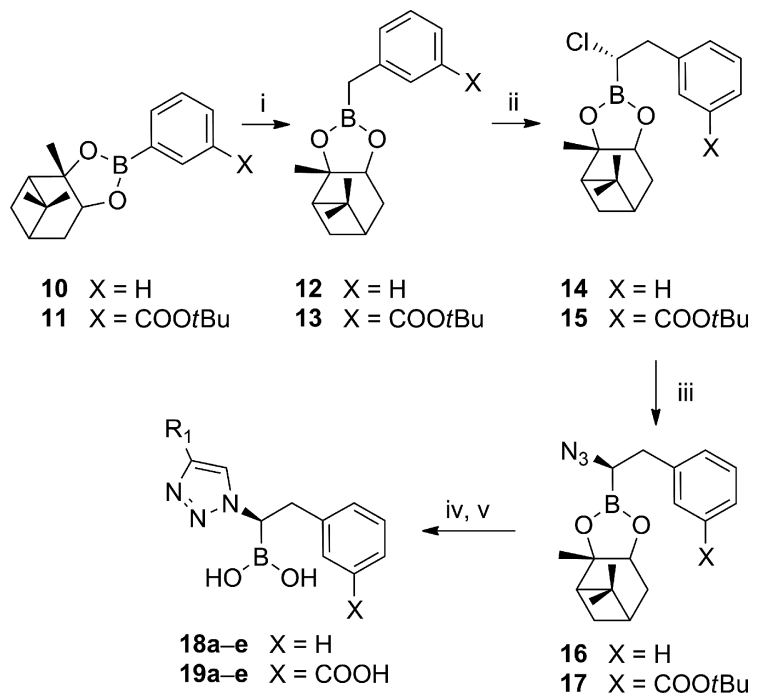

Scheme 3. Stereoselective synthesis of triazolyl analogs of acylboroPhe. (i) $\mathrm{LiCH}_{2} \mathrm{Cl}$, THF, $-80^{\circ} \mathrm{C} \rightarrow$ room temp; (ii) $\mathrm{LiCHCl}_{2}$, $\mathrm{THF},-100^{\circ} \mathrm{C} \rightarrow$ room temp.; (iii) $\mathrm{NaN}_{3}$, TBAHS, EtOAc, $\mathrm{H}_{2} \mathrm{O}$, room temp.; (iv) alkyne, $\mathrm{CuSO}_{4}$, sodium ascorbate, $t \mathrm{BuOH}, \mathrm{H}_{2} \mathrm{O}$, room temp.; (v) phenylboronic acid, $\mathrm{HCl}$, acetonitrile, $n$-hexane, room temp.

(+)-Pinanediol boronates $\mathbf{1 0}$ and $\mathbf{1 1}$ were subjected to two consecutive homologation steps: the first with chloromethyllithium for methylene insertion to $\mathbf{1 2}$ and $\mathbf{1 3}$, and the second with dichloromethyllithium to introduce the halogenated carbon atom (14 and 15). ${ }^{[17]}$ Chlorine substitution with sodium azide under phase transfer conditions afforded 16 and 17. These key azido intermediates were then subjected to click reaction and deprotection to triazolyl analogs of acyl-boroPhe 18a-18e and 19a-19e (Table 2, Entries 6$15)$.

The results reported in Table 2 indicate that the described procedure is reproducible and highly efficient, and affords, in all cases, the expected triazolylmethylboronic acid in moderate to good overall yields as pure and stable solids, which can be stored for months at $+4{ }^{\circ} \mathrm{C}$. The rate of the $\mathrm{CuAAC}$ reaction is not affected by the structure of the azidomethylboronate, but only by the electronic density of the alkyne partner: for a given alkyne, reaction times are consistent for both primary (intermediate 2) and secondary azides (intermediates 16 and 17). Furthermore, for these latter derivatives cycloaddition reaction proceeds without any change in the diastereoisomeric composition, to eventually afford enantiomerically pure triazolylmethylboronic acids.

\section{Conclusions}

A synthetic procedure for enantiomerically-pure [(1,2,3triazol-1-yl)methyl]boronic acids has been developed. This new scaffold can be obtained through CuAAC reaction between stereoisomerically pure 1-azidoalkylboronates and terminal acetylenes, which are catalyzed by copper sulfate, reduced in situ to $\mathrm{Cu}^{\mathrm{I}}$ by sodium ascorbate in a tert-butanol/water system. Under these conditions, the proximity of the reaction center to the boronic group is not detrimental to the stability of the $s p^{3} \mathrm{C}-\mathrm{B}$ bond to copper(I) catalysis, which further expands the functional group compatibility in $\mathrm{CuAAC}$ reaction beyond what is already known. Application of powerful click chemistry to boronates enables many analogs to be synthesized quickly. Given the importance of $\alpha$-amidomethylboronic acids as proteases inhibitors, this efficient access to a new bioisosteric scaffold could promote further exploration of boronates as a promising class of biological active compounds.

\section{Experimental Section}

General Methods: All reactions were performed under an argon atmosphere with oven-dried glassware and dry solvents. Dry THF was obtained by standard methods and freshly distilled under an argon atmosphere from sodium benzophenone ketyl prior to use. All of the reagents were used as purchased from commercial suppliers without further purification. The $-100^{\circ} \mathrm{C}$ bath was prepared by addition of liquid nitrogen to a pre-cooled $\left(-78^{\circ} \mathrm{C}\right)$ mixture of $1: 1$ ethanol/methanol. Preloaded $(0.25 \mathrm{~mm})$ glass supported silica gel plates (Kieselgel 60, Merck) were used for TLC analysis, and compounds were visualized by exposure to UV light and by dipping the plates in $\mathrm{Ce}\left(\mathrm{SO}_{4}\right) \cdot 4 \mathrm{H}_{2} \mathrm{O}(1 \%),\left(\mathrm{NH}_{4}\right)_{6} \mathrm{Mo}_{7} \mathrm{O}_{24} \cdot 4 \mathrm{H}_{2} \mathrm{O}(2.5 \%)$ in sulfuric acid $(10 \%)$ followed by heating on a hot plate. Melting points were measured in open capillary tubes with a Stuart SMP30 Melting Point apparatus. Optical rotations were determined at $+20^{\circ} \mathrm{C}$ with a Perkin-Elmer 241 polarimeter and are expressed in $10^{-1} \mathrm{deg} \mathrm{cm}^{2} \mathrm{~g}^{-1}$. ${ }^{1} \mathrm{H}$ and ${ }^{13} \mathrm{C}$ NMR spectra were recorded with a Bruker Avance- $400 \mathrm{MHz}$ spectrometer. Chemical shifts were calibrated to the residual signals of the deuterated solvent. ${ }^{[20]}$ Multiplicity is given as $\mathrm{s}=$ singlet, $\mathrm{d}=$ doublet, $\mathrm{t}=$ triplet, $\mathrm{q}=$ quartet, $\mathrm{m}$ $=$ multiplet, br. $=$ broad signal. Two-dimensional NMR techniques (COSY, HMBC, HSQC) were used to aid in the assignment of signals in ${ }^{1} \mathrm{H}$ and ${ }^{13} \mathrm{C}$ spectra. In the ${ }^{13} \mathrm{C}$ spectra the signal of the boron-bearing carbon atom tends to be broad, often below the detection limit; however, its resonance was always unambiguously determined by HSQC. The triazole ring carbon signals are often below the detection limit; when possible these were determined by HSQC and HMBC. High-resolution mass spectra were recorded with an Agilent Technologies 6520 Accurate-Mass Q-TOF LC/MS. Elemental analyses were performed with a Carlo Erba Elemental Analyzer 1110.

General Procedure for CuAAC Between Azidomethylboronates and Terminal Acetylenes: Azidomethylboronate $(1.00 \mathrm{mmol})$, the se- 
lected terminal alkyne $(1.50 \mathrm{mmol})$, copper sulfate solution $(50 \mathrm{mg} /$ $\mathrm{mL}, 0.05 \mathrm{mmol})$ and sodium ascorbate $(0.20 \mathrm{mmol})$ were dissolved in a mixture of tert-butanol and water $(1: 1 ; 2.0 \mathrm{~mL}$ of each). The reaction was stirred at room temperature for $2-16 \mathrm{~h}$ (as specified in each case), until the azido boronate disappeared as monitored by TLC. The mixture was then partitioned between ethyl acetate $(20 \mathrm{~mL})$, water $(10 \mathrm{~mL})$ and saturated $\mathrm{NaCl}(8 \mathrm{~mL})$, and the aqueous phase extracted with ethyl acetate $(2 \times 20 \mathrm{~mL})$. The combined organic phases were washed with brine $(15 \mathrm{~mL})$, dried with $\mathrm{Na}_{2} \mathrm{SO}_{4}$, filtered, and concentrated in vacuo, to afford the expected [(1,2,3-triazol-1-yl)methyl]boronate.

(+)-Pinanediyl [(4-Ethoxycarbonyl-1,2,3-triazol-1-yl)methyl]boronate (3a): Yellow viscous oil (reaction time $2 \mathrm{~h}, 97 \%$ ). $[a]_{\mathrm{D}}^{20}=+13.6$ $\left(c=1.3, \mathrm{CHCl}_{3}\right) .{ }^{1} \mathrm{H} \mathrm{NMR}\left(400 \mathrm{MHz}, \mathrm{CDCl}_{3}\right): \delta=0.76(\mathrm{~s}, 3 \mathrm{H}$, pinanyl $\left.\mathrm{CH}_{3}\right), 1.03\left(\mathrm{~d}, J=11.1 \mathrm{~Hz}, 1 \mathrm{H}\right.$, pinanyl $\left.H_{\text {endo }}\right), 1.21(\mathrm{~s}, 3$ $\mathrm{H}$, pinanyl $\left.\mathrm{CH}_{3}\right), 1.32\left(\mathrm{t}, \mathrm{J}=7.0 \mathrm{~Hz}, 3 \mathrm{H}, \mathrm{OCH}_{2} \mathrm{CH}_{3}\right), 1.35$ (s, 3 $\mathrm{H}$, pinanyl $\mathrm{CH}_{3}$ ), $1.77-2.30$ (m, $5 \mathrm{H}$, pinanyl protons), 4.20 (s, 2 $\left.\mathrm{H}, \mathrm{CH}_{2} \mathrm{~B}\right), 4.29-4.35\left(\mathrm{~m}, 3 \mathrm{H}, \mathrm{CHOB}, \mathrm{OCH}_{2} \mathrm{CH}_{3}\right), 8.19(\mathrm{~s}, 1 \mathrm{H}$, $\left.\mathrm{CH}_{\text {triaz }}\right)$ ppm. ${ }^{13} \mathrm{C}$ NMR $\left(100 \mathrm{MHz}, \mathrm{CDCl}_{3}\right): \delta=14.2,23.8,26.4$, 291 26.9, 28.3, 34.9, 35.9 (br., CB), 38.1, 39.2, 51.0, 61.0, 78.9, 87.7, $128.6,139.9,160.9 \mathrm{ppm}$. HRMS (ESI-TOF) $\mathrm{m} / \mathrm{z}$ : calcd. for $\mathrm{C}_{16} \mathrm{H}_{25} \mathrm{BN}_{3} \mathrm{O}_{4}[\mathrm{M}+\mathrm{H}]^{+}$334.1936; found 334.1938.

(+)-Pinanediyl [(4-Carboxy-1,2,3-triazol-1-yl)methyl]boronate (3b): White solid (reaction time $2 \mathrm{~h}, 85 \%$ ), m.p. $110-113{ }^{\circ} \mathrm{C} \mathrm{dec}$. $[\alpha]_{\mathrm{D}}^{20}=$ $+16.6\left(c=1.3, \mathrm{CHCl}_{3}\right) .{ }^{1} \mathrm{H}$ NMR $\left(400 \mathrm{MHz}, \mathrm{CDCl}_{3}\right): \delta=0.82(\mathrm{~s}$, $3 \mathrm{H}$, pinanyl $\left.\mathrm{CH}_{3}\right), 1.08\left(\mathrm{~d}, J=11.1 \mathrm{~Hz}, 1 \mathrm{H}\right.$, pinanyl $\left.H_{\text {endo }}\right), 1.27$ (s, $3 \mathrm{H}$, pinanyl $\left.\mathrm{CH}_{3}\right), 1.42\left(\mathrm{~s}, 3 \mathrm{H}\right.$, pinanyl $\left.\mathrm{CH}_{3}\right), 1.84-2.37(\mathrm{~m}, 5$ $\mathrm{H}$, pinanyl protons), 4.29 (s, $\left.2 \mathrm{H}, \mathrm{CH}_{2} \mathrm{~B}\right), 4.39$ (dd, $J=8.7,4.4 \mathrm{~Hz}$, $1 \mathrm{H}, \mathrm{CHOB}), 8.37$ (s, $\left.1 \mathrm{H}, \mathrm{C} H_{\text {triaz }}\right), 9.57(\mathrm{~s}, 1 \mathrm{H}, \mathrm{COOH}) \mathrm{ppm} .{ }^{13} \mathrm{C}$ 301 NMR (100 MHz, $\left.\mathrm{CDCl}_{3}\right): \delta=24.1,26.6,27.1,28.5,35.1,36.3$ (br., CB), 38.3, 39.4, 51.1, 79.2, 88.0, 129.5, 139.3, 164.1 ppm. HRMS (ESI-TOF) $m / z$ : calcd. for $\mathrm{C}_{14} \mathrm{H}_{21} \mathrm{BN}_{3} \mathrm{O}_{4}[\mathrm{M}+\mathrm{H}]^{+} 306.1622$; found 306.1624 .

(+)-Pinanediyl [(4-Phenyl-1,2,3-triazol-1-yl)methyl|boronate (3c): 306 Yellow viscous oil (reaction time $16 \mathrm{~h}, 99 \%)$. $[a]_{\mathrm{D}}^{20}=+13.0(c=2.2$, $\mathrm{CHCl}_{3}$ ). ${ }^{1} \mathrm{H}$ NMR $\left(400 \mathrm{MHz}, \mathrm{CDCl}_{3}\right): \delta=0.83$ (s, $3 \mathrm{H}$, pinanyl $\left.\mathrm{CH}_{3}\right), 1.15\left(\mathrm{~d}, J=11.1 \mathrm{~Hz}, 1 \mathrm{H}\right.$, pinanyl $\left.H_{\text {endo }}\right), 1.28(\mathrm{~s}, 3 \mathrm{H}$, pinanyl $\left.\mathrm{CH}_{3}\right), 1.43\left(\mathrm{~s}, 3 \mathrm{H}\right.$, pinanyl $\left.\mathrm{CH}_{3}\right), 1.85-2.37(\mathrm{~m}, 5 \mathrm{H}$, pinanyl protons), $4.24\left(\mathrm{~s}, 2 \mathrm{H}, \mathrm{CH}_{2} \mathrm{~B}\right), 4.39(\mathrm{dd}, J=8.7,1.8 \mathrm{~Hz}, 1 \mathrm{H}$,

$311 \mathrm{CHOB}), 7.29\left(\mathrm{t}, J=7.4 \mathrm{~Hz}, 1 \mathrm{H}, \mathrm{H}_{\text {arom }}\right), 7.39(\mathrm{t}, J=7.9 \mathrm{~Hz}, 2 \mathrm{H}$, $\left.\mathrm{H}_{\text {arom }}\right), 7.82$ (d, $\left.J=7.4 \mathrm{~Hz}, 2 \mathrm{H}, \mathrm{H}_{\text {arom }}\right), 7.89$ (s, $\left.1 \mathrm{H}, \mathrm{CH}_{\text {triaz }}\right) \mathrm{ppm}$. ${ }^{13} \mathrm{C}$ NMR $\left(100 \mathrm{MHz}, \mathrm{CDCl}_{3}\right): \delta=24.0,26.5,27.0,28.5,35.1,35.6$ (br., CB), 38.2, 39.4, 51.1, 78.9, 87.5, 121.0, 125.7, 127.9, 128.8, 131.0, $147.6 \mathrm{ppm}$. HRMS (ESI-TOF) $\mathrm{m} / \mathrm{z}$ : calcd. for $\mathrm{C}_{19} \mathrm{H}_{25} \mathrm{BN}_{3} \mathrm{O}_{2}$

$316[\mathrm{M}+\mathrm{H}]^{+}$338.2038; found 338.2031.

(+)-Pinanediyl [(4-Thiophen-3-yl-1,2,3-triazol-1-yl)methyl]boronate (3d): Yellow viscous oil (reaction time $16 \mathrm{~h}, 92 \%)$. $[\alpha]_{\mathrm{D}}^{20}=+10.2(c$ $\left.=0.9, \mathrm{CHCl}_{3}\right) .{ }^{1} \mathrm{H}$ NMR $\left(400 \mathrm{MHz}, \mathrm{CDCl}_{3}\right): \delta=0.83(\mathrm{~s}, 3 \mathrm{H}$, pinanyl $\left.\mathrm{CH}_{3}\right), 1.14\left(\mathrm{~d}, J=11.1 \mathrm{~Hz}, 1 \mathrm{H}\right.$, pinanyl $\left.H_{\text {endo }}\right), 1.28$ (s, 3

$321 \mathrm{H}$, pinanyl $\left.\mathrm{CH}_{3}\right), 1.43\left(\mathrm{~s}, 3 \mathrm{H}\right.$, pinanyl $\left.\mathrm{CH}_{3}\right), 1.85-2.37(\mathrm{~m}, 5 \mathrm{H}$, pinanyl protons), $4.23\left(\mathrm{~s}, 2 \mathrm{H}, \mathrm{CH}_{2} \mathrm{~B}\right), 4.38$ (dd, $J=8.8,1.7 \mathrm{~Hz}, 1$ H, CHOB), $7.34(\mathrm{dd}, J=5.0,2.9 \mathrm{~Hz}, 1 \mathrm{H}, \mathrm{CHCHS}), 7.45(\mathrm{dd}, J$ $=5.0,1.1 \mathrm{~Hz}, 1 \mathrm{H}, \mathrm{CHCHS}), 7.64(\mathrm{dd}, J=2.9,1.1 \mathrm{~Hz}, 1 \mathrm{H}$, $\mathrm{CCHS}), 7.79$ (s, $\left.1 \mathrm{H}, \mathrm{CH}_{\text {triaz }}\right)$ ppm. ${ }^{13} \mathrm{C} \mathrm{NMR}\left(100 \mathrm{MHz}, \mathrm{CDCl}_{3}\right)$ : $\delta=24.0,26.6,27.0,28.5,35.1,35.8$ (br., CB), 38.2, 39.4, 51.1, 78.9, $87.6,120.8,120.9,126.0,126.1,132.3,143.8$ ppm. HRMS (ESITOF) $m / z$ : calcd. for $\mathrm{C}_{17} \mathrm{H}_{23} \mathrm{BN}_{3} \mathrm{O}_{2} \mathrm{~S}[\mathrm{M}+\mathrm{H}]^{+} 344.1602$; found 344.1610 .

(+)-Pinanediyl [(4-Phenoxymethyl-1,2,3-triazol-1-yl)methyl]boronate (3e): Yellow viscous oil (reaction time $8 \mathrm{~h}, 97 \%$ ). $[\alpha]_{\mathrm{D}}^{20}=+13.1$ $\left(c=2.1, \mathrm{CHCl}_{3}\right) .{ }^{1} \mathrm{H}$ NMR $\left(400 \mathrm{MHz}, \mathrm{CDCl}_{3}\right): \delta=0.85(\mathrm{~s}, 3 \mathrm{H}$, pinanyl $\left.\mathrm{CH}_{3}\right), 1.13\left(\mathrm{~d}, J=11.1 \mathrm{~Hz}, 1 \mathrm{H}\right.$, pinanyl $\left.H_{\text {endo }}\right), 1.30(\mathrm{~s}, 3$
$\mathrm{H}$, pinanyl $\left.\mathrm{CH}_{3}\right), 1.43\left(\mathrm{~s}, 3 \mathrm{H}\right.$, pinanyl $\left.\mathrm{CH}_{3}\right), 1.85-2.39(\mathrm{~m}, 5 \mathrm{H}$, pinanyl protons), $4.22\left(\mathrm{~s}, 2 \mathrm{H}, \mathrm{CH}_{2} \mathrm{~B}\right), 4.39$ (dd, $J=8.8,1.8 \mathrm{~Hz}, 1$ $\mathrm{H}, \mathrm{CHOB}), 5.21\left(\mathrm{~s}, 2 \mathrm{H}, \mathrm{OCH}_{2}\right), 6.96\left(\mathrm{t}, J=7.3 \mathrm{~Hz}, 1 \mathrm{H}, \mathrm{H}_{\text {arom }}\right)$, $7.00\left(\mathrm{~d}, J=8.7 \mathrm{~Hz}, 2 \mathrm{H}, \mathrm{H}_{\mathrm{arom}}\right), 7.28(\mathrm{dd}, J=8.7,7.3 \mathrm{~Hz}, 2 \mathrm{H}$ $\left.\mathrm{H}_{\text {arom}}\right), 7.77$ (s, $\left.1 \mathrm{H}, \mathrm{CH}_{\text {triaz }}\right) \mathrm{ppm} .{ }^{13} \mathrm{C} \mathrm{NMR}\left(100 \mathrm{MHz}, \mathrm{CDCl}_{3}\right)$ : $\delta=24.0,26.5,27.0,28.5,35.1,35.8$ (br., $C \mathrm{~B}$ ), 38.2, 39.4, 51.1, 62.1, $78.9,87.5,114.9,121.2,124.1,129.5,143.9,158.4$ ppm. HRMS (ESI-TOF) $m / z$ : calcd. for $\mathrm{C}_{20} \mathrm{H}_{27} \mathrm{BN}_{3} \mathrm{O}_{3}[\mathrm{M}+\mathrm{H}]^{+} 368.2144$; found 368.2139 .

General Procedure for Deprotection of Pinanediyl Boronates Through Transesterification: To a solution of [(1,2,3-triazol-1-yl) methyl]boronate $(0.50 \mathrm{mmol})$ in $\mathrm{CH}_{3} \mathrm{CN}(3 \mathrm{~mL}), \mathrm{HCl}(3 \mathrm{M}$ aqueous solution, $1.50 \mathrm{mmol})$, phenylboronic acid $(0.47 \mathrm{mmol})$, and $n$-hexane $(3 \mathrm{~mL})$ were sequentially added and the resulting biphasic solution was vigorously stirred. After $30 \mathrm{~min}$ the $n$-hexane layer, which contained the pinanediol phenylboronate, was removed and fresh $n$-hexane $(3 \mathrm{~mL})$ was added. This last step was repeated several times until a TLC analysis of the $n$-hexane layer revealed no phenylboronate production (total reaction time $3 \mathrm{~h}$ ). The acetonitrile phase was then concentrated and the crude product was recrystallized from acetonitrile to afford [(1,2,3-triazol-1-yl)methyl]boronic acid.

The enantiomeric purity of chiral boronic acids was checked by reconversion into their pinanediol esters. Final compounds $\mathbf{9 a}-\mathbf{9 e}$, 18a-18e, and 19a-19e were allowed to react with an equimolar amount of (+)-pinanediol in anhydrous THF: the NMR spectra of the crude products displayed the presence of a single diastereoisomer, which proves that no epimerization occurred during the transesterification reaction.

[(4-Ethoxycarbonyl-1,2,3-triazol-1-yl)methyl]boronic Acid (4a): White solid $(80 \%)$, m.p. $123-125{ }^{\circ} \mathrm{C}$ dec. ${ }^{1} \mathrm{H}$ NMR $(400 \mathrm{MHz}$, $\left.\mathrm{CD}_{3} \mathrm{OD}\right): \delta=1.38\left(\mathrm{t}, J=7.1 \mathrm{~Hz}, 3 \mathrm{H}, \mathrm{OCH}_{2} \mathrm{CH}_{3}\right), 4.27(\mathrm{~s}, 2 \mathrm{H}$, $\left.\mathrm{CH}_{2} \mathrm{~B}\right), 4.38$ (q, $\left.J=7.1 \mathrm{~Hz}, 2 \mathrm{H}, \mathrm{OCH}_{2} \mathrm{CH}_{3}\right), 8.38(\mathrm{~s}, 1 \mathrm{H}$, $\left.\mathrm{C}_{\text {triaz }}\right)$ ppm. ${ }^{13} \mathrm{C}$ NMR $\left(100 \mathrm{MHz}, \mathrm{CD}_{3} \mathrm{OD}\right): \delta=14.6,39.3$ (br., CB), 62.1, 130.5, $140.4 \mathrm{ppm}, \mathrm{COOEt}$ not seen. HRMS (ESI-TOF) $\mathrm{m} / z$ : calcd. for $\mathrm{C}_{6} \mathrm{H}_{11} \mathrm{BN}_{3} \mathrm{O}_{4}[\mathrm{M}+\mathrm{H}]^{+} 200.0838$; found 200.0840 .

[(4-Carboxy-1,2,3-triazol-1-yl)methyl|boronic Acid (4b): White solid (80\%), m.p. $236-240{ }^{\circ} \mathrm{C}$ dec. ${ }^{1} \mathrm{H}$ NMR (400 MHz, $\left.\mathrm{CD}_{3} \mathrm{OD}\right): \delta=$ $4.26\left(\mathrm{~s}, 2 \mathrm{H}, \mathrm{CH}_{2} \mathrm{~B}\right), 8.39\left(\mathrm{~s}, 1 \mathrm{H}, \mathrm{CH}_{\text {triaz }}\right) \mathrm{ppm} .{ }^{13} \mathrm{C} \mathrm{NMR}$ (100 MHz, $\mathrm{CD}_{3} \mathrm{OD}$ ): $\delta=39.9$ (br., $C \mathrm{~B}$ ), 130.7, 140.6, $163.3 \mathrm{ppm}$ HRMS (ESI-TOF) $m / z$ : calcd. for $\mathrm{C}_{4} \mathrm{H}_{7} \mathrm{BN}_{3} \mathrm{O}_{4}[\mathrm{M}+\mathrm{H}]^{+} 172.0525$; found 172.0518

[(4-Phenyl-1,2,3-triazol-1-yl)methyl|boronic Acid (4c): Grey solid (98\%), m.p. $122-124{ }^{\circ} \mathrm{C}$ dec. ${ }^{1} \mathrm{H}$ NMR $\left(400 \mathrm{MHz}, \mathrm{CD}_{3} \mathrm{OD}\right): \delta=$ $4.48\left(\mathrm{~s}, 2 \mathrm{H}, \mathrm{CH}_{2} \mathrm{~B}\right), 7.56-7.60\left(\mathrm{~m}, 3 \mathrm{H}, \mathrm{H}_{\text {arom }}\right), 7.83$ (dd, $J=7.8$, $\left.1.7 \mathrm{~Hz}, 2 \mathrm{H}, \mathrm{H}_{\text {arom }}\right), 8.78\left(\mathrm{~s}, 1 \mathrm{H}, \mathrm{CH}_{\text {triaz }}\right) \mathrm{ppm} .{ }^{13} \mathrm{C} \mathrm{NMR}$ (100 MHz, $\mathrm{CD}_{3} \mathrm{OD}$ ): $\delta=42.6$ (br., $C \mathrm{~B}$ ), 126.0, 126.8, 127.6, 130.7, 131.9, $144.7 \mathrm{ppm}$. HRMS (ESI-TOF) $\mathrm{m} / z$ : calcd. for $\mathrm{C}_{9} \mathrm{H}_{11} \mathrm{BN}_{3} \mathrm{O}_{2}$ $[\mathrm{M}+\mathrm{H}]^{+}$204.0941; found 204.0940.

[(4-Thiophen-3-yl-1,2,3-triazol-1-yl)methyl]boronic Acid (4d): White solid (100\%), m.p. $170-172{ }^{\circ} \mathrm{C}$ dec. ${ }^{1} \mathrm{H}$ NMR $\left(400 \mathrm{MHz}, \mathrm{CD}_{3} \mathrm{OD}\right)$ : $\delta=4.47\left(\mathrm{~s}, 2 \mathrm{H}, \mathrm{CH}_{2} \mathrm{~B}\right), 7.55(\mathrm{dd}, J=5.1,1.1 \mathrm{~Hz}, 1 \mathrm{H}, \mathrm{CHCHS})$, $7.69(\mathrm{dd}, J=5.1,2.8 \mathrm{~Hz}, 1 \mathrm{H}, \mathrm{CHCHS}), 8.08(\mathrm{dd}, J=2.8,1.1 \mathrm{~Hz}$, $1 \mathrm{H}, \mathrm{CCHS}), 8.70\left(\mathrm{~s}, 1 \mathrm{H}, \mathrm{C} H_{\text {triaz }}\right)$ ppm. ${ }^{13} \mathrm{C} \mathrm{NMR}(100 \mathrm{MHz}$, $\mathrm{CD}_{3} \mathrm{OD}$ ): $\delta=42.7$ (br., $C \mathrm{CB}$ ), 126.4, 126.5, 126.6, 127.0, 129.6, $140.4 \mathrm{ppm}$. HRMS (ESI-TOF) $\mathrm{m} / z$ : calcd. for $\mathrm{C}_{7} \mathrm{H}_{9} \mathrm{BN}_{3} \mathrm{O}_{2} \mathrm{~S}[\mathrm{M}+$ $\mathrm{H}]^{+}$210.0504; found 210.0498

[(4-Phenoxymethyl-1,2,3-triazol-1-yl)methyl]boronic Acid (4e): White solid (100\%), m.p. $128-131{ }^{\circ} \mathrm{C}$ dec. ${ }^{1} \mathrm{H}$ NMR $(400 \mathrm{MHz}$, $\mathrm{CD}_{3} \mathrm{OD}$ ): $\delta=4.44$ (s, $2 \mathrm{H}, \mathrm{CH}_{2} \mathrm{~B}$ ), 5.33 (s, $2 \mathrm{H}, \mathrm{OCH}_{2}$ ), 7.01 (t, $J$ $\left.=7.4 \mathrm{~Hz}, 1 \mathrm{H}, \mathrm{H}_{\text {arom }}\right), 7.05\left(\mathrm{dd}, J=8.7,0.8 \mathrm{~Hz}, 2 \mathrm{H}, \mathrm{H}_{\text {arom }}\right), 7.32$ 
$\left(\mathrm{dd}, J=8.7,7.4 \mathrm{~Hz}, 2 \mathrm{H}, \mathrm{H}_{\text {arom }}\right), 8.48\left(\mathrm{~s}, 1 \mathrm{H}, \mathrm{C} H_{\text {triaz }}\right)$ ppm. ${ }^{13} \mathrm{C}$ NMR (100 MHz, CD 3 OD): $\delta=42.5$ (br., CB), 60.4, 116.0, 123.1, $129.0,130.8,141.5,159.0 \mathrm{ppm}$. HRMS (ESI-TOF) $\mathrm{m} / \mathrm{z}$ : calcd. for $\mathrm{C}_{10} \mathrm{H}_{13} \mathrm{BN}_{3} \mathrm{O}_{3}[\mathrm{M}+\mathrm{H}]^{+}$234.1046; found 234.1048.

(+)-Pinanediyl [(1R)-1-Azido-3-methylbutyl]boronate (7): To a solution of $6^{[21]}(800 \mathrm{mg}, 2.81 \mathrm{mmol})$ in ethyl acetate $(10 \mathrm{~mL})$, sodium azide $(1.83 \mathrm{~g}, 28.1 \mathrm{mmol})$, tetrabutylammonium hydrogensulfate (475 mg, $1.40 \mathrm{mmol})$, and water $(24 \mathrm{~mL})$ were added and the system was vigorously stirred at room temperature overnight. The mixture was then diluted with saturated ammonium chloride/water $(1: 1,40 \mathrm{~mL})$ and extracted twice with light petroleum $(60 \mathrm{~mL}$, $30 \mathrm{~mL}$ ). The combined organic fractions were washed again with saturated ammonium chloride/water $(1: 1,20 \mathrm{~mL})$, dried with $\mathrm{Na}_{2} \mathrm{SO}_{4}$, and filtered. Removal of solvent in vacuo afforded 7 as a colorless oil (794 mg, 97\% yield). $[a]_{\mathrm{D}}^{20}=-7.13\left(c=1.7, \mathrm{CH}_{2} \mathrm{Cl}_{2}\right)$. ${ }^{1} \mathrm{H}$ NMR (400 MHz, $\left.\mathrm{CDCl}_{3}\right): \delta=0.83\left(\mathrm{~s}, 3 \mathrm{H}\right.$, pinanyl $\left.\mathrm{CH}_{3}\right), 0.92$ $\left[\mathrm{d}, J=6.6 \mathrm{~Hz}, 3 \mathrm{H}, \mathrm{CH}\left(\mathrm{CH}_{3}\right)_{2}\right], 0.93\left[\mathrm{~d}, J=6.6 \mathrm{~Hz}, 3 \mathrm{H}, \mathrm{CH}\left(\mathrm{CH}_{3}\right)_{2}\right.$ ], 1.09 (d, $J=11.0 \mathrm{~Hz}, 1 \mathrm{H}$, pinanyl $\left.H_{\text {endo }}\right), 1.28$ (s, $3 \mathrm{H}$, pinanyl $\left.\mathrm{CH}_{3}\right), 1.40\left(\mathrm{~s}, 3 \mathrm{H}\right.$, pinanyl $\left.\mathrm{CH}_{3}\right), 1.45$ (ddd, $J=14.0,8.5,5.4 \mathrm{~Hz}$, $1 \mathrm{H}, \mathrm{BCHCH}_{2}$ ), 1.63 (ddd, $J=14.0,10.0,5.4 \mathrm{~Hz}, 1 \mathrm{H}, \mathrm{BCHCH}_{2}$ ), 1.76-1.83 [m, $\left.1 \mathrm{H}, \mathrm{CH}\left(\mathrm{CH}_{3}\right)_{2}\right], 1.85-2.37$ (m, $5 \mathrm{H}$, pinanyl protons), 3.11 (dd, $J=10.0,5.4 \mathrm{~Hz}, 1 \mathrm{H}, \mathrm{BCH}), 4.34$ (dd, $J=8.8$, $1.8 \mathrm{~Hz}, 1 \mathrm{H}, \mathrm{CHOB}) \mathrm{ppm} .{ }^{13} \mathrm{C}$ NMR $\left(100 \mathrm{MHz}, \mathrm{CDCl}_{3}\right): \delta=21.7$, 23.1, 24.0, 25.7, 26.6, 27.1, 28.6, 35.4, 38.2, 39.3, 39.5, 46.4 (br., CB), 51.2, 78.5, 86.9 ppm. $\mathrm{C}_{15} \mathrm{H}_{26} \mathrm{BN}_{3} \mathrm{O}_{2}$ (291.20): calcd. C 61.87, $\mathrm{H} 9.00, \mathrm{~N} 14.43$; found $\mathrm{C} 61.66, \mathrm{H} 9.21, \mathrm{~N} 14.29$

(+)-Pinanediyl [(1R)-1-(4-Ethoxycarbonyl-1,2,3-triazol-1-yl)-3methylbutyl|boronate (8a): According to the general procedure reported above, CuAAC reaction between azido boronate 7 and ethyl propiolate (reaction time $2 \mathrm{~h}$ ) afforded 8a as a yellow viscous oil (reaction time $2 \mathrm{~h}, 89 \%) \cdot[a]_{\mathrm{D}}^{20}=+25.0\left(c=0.9, \mathrm{CHCl}_{3}\right) \cdot{ }^{1} \mathrm{H} \mathrm{NMR}$ $\left(400 \mathrm{MHz}, \mathrm{CDCl}_{3}\right): \delta=0.80\left(\mathrm{~s}, 3 \mathrm{H}\right.$, pinanyl $\left.\mathrm{CH}_{3}\right), 0.83[\mathrm{~d}, J=$ $\left.6.6 \mathrm{~Hz}, 3 \mathrm{H}, \mathrm{CH}\left(\mathrm{CH}_{3}\right)_{2}\right], 0.90\left[\mathrm{~d}, J=6.5 \mathrm{~Hz}, 3 \mathrm{H}, \mathrm{CH}\left(\mathrm{CH}_{3}\right)_{2}\right], 1.03$ (d, $J=11.1 \mathrm{~Hz}, 1 \mathrm{H}$, pinanyl $\left.H_{\text {endo }}\right), 1.25\left(\mathrm{~s}, 3 \mathrm{H}\right.$, pinanyl $\left.\mathrm{CH}_{3}\right)$, 1.28-1.34 [m, $\left.1 \mathrm{H}, \mathrm{CH}\left(\mathrm{CH}_{3}\right)_{2}\right], 1.38\left(\mathrm{~s}, 3 \mathrm{H}\right.$, pinanyl $\left.\mathrm{CH}_{3}\right), 1.38(\mathrm{t}$, $\left.J=7.0 \mathrm{~Hz}, 3 \mathrm{H}, \mathrm{OCH}_{2} \mathrm{CH}_{3}\right), 1.71-2.34(\mathrm{~m}, 7 \mathrm{H}$, pinanyl protons,

$\left.431 \mathrm{BCHCH}_{2}\right), 4.32(\mathrm{~d}, J=8.7 \mathrm{~Hz}, 1 \mathrm{H}, \mathrm{CHOB}), 4.39$ (q, $J=7.0 \mathrm{~Hz}$, $\left.2 \mathrm{H}, \mathrm{OCH}_{2} \mathrm{CH}_{3}\right), 4.60$ (dd, $\left.J=10.3,5.5 \mathrm{~Hz}, 1 \mathrm{H}, \mathrm{BCH}\right), 8.16$ (s, 1 $\left.\mathrm{H}, \mathrm{CH}_{\text {triaz }}\right) \mathrm{ppm} .{ }^{13} \mathrm{C} \mathrm{NMR}\left(100 \mathrm{MHz} \mathrm{CDCl}_{3}\right): \delta=14.4,21.4$, 22.8, 24.0, 25.0, 26.5, 27.0, 28.5, 35.2, 38.2, 39.4, 41.4, 47.1 (br., CB), 51.1, 61.2, 78.9, 87.6, 127.5, 140.1, 161.2 ppm. HRMS (ESI-

436 TOF) $m / z$ : calcd. for $\mathrm{C}_{20} \mathrm{H}_{33} \mathrm{BN}_{3} \mathrm{O}_{4}[\mathrm{M}+\mathrm{H}]^{+} 390.2562$; found 390.2563

(+)-Pinanediyl I(1R)-1-(4-Carboxy-1,2,3-triazol-1-yl)-3-methylbutyl]boronate (8b): Yellow viscous oil (reaction time $2 \mathrm{~h}, 91 \%$ ). $[\alpha]_{\mathrm{D}}^{20}$ $=+19.5\left(c=1.4, \mathrm{CHCl}_{3}\right) .{ }^{1} \mathrm{H} \mathrm{NMR}\left(400 \mathrm{MHz}, \mathrm{CDCl}_{3}\right): \delta=0.82$ (s, $3 \mathrm{H}$, pinanyl $\left.\mathrm{CH}_{3}\right), 0.86\left[\mathrm{~d}, J=6.4 \mathrm{~Hz}, 3 \mathrm{H}, \mathrm{CH}\left(\mathrm{CH}_{3}\right)_{2}\right], 0.94$ $\left[\mathrm{d}, J=6.3 \mathrm{~Hz}, 3 \mathrm{H}, \mathrm{CH}\left(\mathrm{CH}_{3}\right)_{2}\right], 1.04(\mathrm{~d}, J=11.1 \mathrm{~Hz}, 1 \mathrm{H}$, pinanyl $\left.H_{\text {endo }}\right), 1.28$ (s, $3 \mathrm{H}$, pinanyl $\left.\mathrm{CH}_{3}\right), 1.32-1.37$ [m, $\left.1 \mathrm{H}, \mathrm{CH}\left(\mathrm{CH}_{3}\right)_{2}\right]$, $1.41\left(\mathrm{~s}, 3 \mathrm{H}\right.$, pinanyl $\left.\mathrm{CH}_{3}\right), 1.83-2.37$ (m, $7 \mathrm{H}$, pinanyl protons, $\left.\mathrm{BCHCH} H_{2}\right), 4.35(\mathrm{dd}, J=8.7,1.5 \mathrm{~Hz}, 1 \mathrm{H}, \mathrm{CHOB}), 4.65$ (dd, $J=$

$44610.3,5.5 \mathrm{~Hz}, 1 \mathrm{H}, \mathrm{BCH}), 8.33$ (s, $\left.1 \mathrm{H}, \mathrm{CH}_{\text {triaz }}\right), 8.74$ (br., $1 \mathrm{H}$, $\mathrm{COO} H)$ ppm. ${ }^{13} \mathrm{C}$ NMR $\left(100 \mathrm{MHz}, \mathrm{CDCl}_{3}\right): \delta=21.4,22.9,24.0$, 25.1, 26.5, 27.1, 28.5, 35.2, 38.3, 39.4, 41.4, 47.7 (br., CB), 51.1, 79.0, 87.8, 128.3, 139.4, 164.0 ppm. HRMS (ESI-TOF) $\mathrm{m} / \mathrm{z}$ : calcd. for $\mathrm{C}_{18} \mathrm{H}_{29} \mathrm{BN}_{3} \mathrm{O}_{4}[\mathrm{M}+\mathrm{H}]^{+} 362.2249$; found 362.2254

451 (+)-Pinanediyl [(1R)-3-Methyl-1-(4-phenyl-1,2,3-triazol-1-yl)butyl]boronate (8c): Yellow viscous oil (reaction time $16 \mathrm{~h}, 81 \%) .[\alpha]_{\mathrm{D}}^{20}=$ $+11.8\left(c=1.8, \mathrm{CHCl}_{3}\right) .{ }^{1} \mathrm{H} \mathrm{NMR}\left(400 \mathrm{MHz}, \mathrm{CDCl}_{3}\right): \delta=0.84(\mathrm{~s}$, $3 \mathrm{H}$, pinanyl $\left.\mathrm{CH}_{3}\right), 0.88\left[\mathrm{~d}, J=6.6 \mathrm{~Hz}, 3 \mathrm{H}, \mathrm{CH}\left(\mathrm{CH}_{3}\right)_{2}\right], 0.97$ [d, $\left.J=6.5 \mathrm{~Hz}, 3 \mathrm{H}, \mathrm{CH}\left(\mathrm{CH}_{3}\right)_{2}\right], 1.13(\mathrm{~d}, J=11.0 \mathrm{~Hz}, 1 \mathrm{H}$, pinanyl
1.42 (s, $3 \mathrm{H}$, pinanyl $\mathrm{CH}_{3}$ ), 1.73-2.38 (m, $7 \mathrm{H}$, pinanyl protons, $\left.\mathrm{BCHCH}_{2}\right), 4.37$ (dd, $\left.J=8.7,1.6 \mathrm{~Hz}, 1 \mathrm{H}, \mathrm{CHOB}\right), 4.60(\mathrm{dd}, J=$ $10.5,5.6 \mathrm{~Hz}, 1 \mathrm{H}, \mathrm{BCH}), 7.31\left(\mathrm{t}, J=7.5 \mathrm{~Hz}, 1 \mathrm{H}, \mathrm{H}_{\text {arom }}\right), 7.41(\mathrm{t}$, $\left.J=7.5 \mathrm{~Hz}, 2 \mathrm{H}, \mathrm{H}_{\text {arom }}\right), 7.85\left(\mathrm{~d}, J=7.5 \mathrm{~Hz}, 2 \mathrm{H}, \mathrm{H}_{\text {arom }}\right), 7.86(\mathrm{~s}$, $\left.1 \mathrm{H}, \mathrm{CH}_{\text {triaz }}\right) \mathrm{ppm} .{ }^{13} \mathrm{C} \mathrm{NMR}\left(100 \mathrm{MHz}, \mathrm{CDCl}_{3}\right): \delta=21.6,23.0$, 24.1, 25.2, 26.6, 27.1, 28.6, 35.3, 38.3, 39.5, 41.6, 46.5 (br., CB), 51.2, 78.9, 87.4, 119.7, 125.8, 128.0, 128.9, 131.2, 147.5 ppm. HRMS (ESI-TOF) $m / z$ : calcd. for $\mathrm{C}_{23} \mathrm{H}_{33} \mathrm{BN}_{3} \mathrm{O}_{2}[\mathrm{M}+\mathrm{H}]^{+}$ 394.2665; found 394.2671.

(+)-Pinanediyl [(1R)-3-Methyl-1-(4-thiophen-3-yl-1,2,3-triazol-1-yl)butyl]boronate (8d): Yellow viscous oil (reaction time $16 \mathrm{~h}, 85 \%$ ). $[a]_{\mathrm{D}}^{20}=+13.1\left(c=1.6, \mathrm{CHCl}_{3}\right) .{ }^{1} \mathrm{H} \mathrm{NMR}\left(400 \mathrm{MHz}, \mathrm{CDCl}_{3}\right): \delta=$ $0.84\left(\mathrm{~s}, 3 \mathrm{H}\right.$, pinanyl $\left.\mathrm{CH}_{3}\right), 0.87\left[\mathrm{~d}, J=6.6 \mathrm{~Hz}, 3 \mathrm{H}, \mathrm{CH}\left(\mathrm{CH}_{3}\right)_{2}\right.$ ], $0.96\left[\mathrm{~d}, J=6.5 \mathrm{~Hz}, 3 \mathrm{H}, \mathrm{CH}\left(\mathrm{CH}_{3}\right)_{2}\right], 1.12(\mathrm{~d}, J=11.0 \mathrm{~Hz}, 1 \mathrm{H}$, pinanyl $\left.H_{\text {endo }}\right), 1.29\left(\mathrm{~s}, 3 \mathrm{H}\right.$, pinanyl $\left.\mathrm{CH}_{3}\right), 1.33-1.38[\mathrm{~m}, 1 \mathrm{H}$, $\mathrm{CH}\left(\mathrm{CH}_{3}\right)_{2}$ ], $1.42\left(\mathrm{~s}, 3 \mathrm{H}\right.$, pinanyl $\left.\mathrm{CH}_{3}\right), 1.72-2.37(\mathrm{~m}, 7 \mathrm{H}$, pinanyl protons, $\left.\mathrm{BCHCH} \mathrm{H}_{2}\right), 4.36(\mathrm{~d}, J=7.2 \mathrm{~Hz}, 1 \mathrm{H}, \mathrm{CHOB}), 4.58$ (dd, $J$ $=10.5,5.5 \mathrm{~Hz}, 1 \mathrm{H}, \mathrm{BCH}), 7.36(\mathrm{dd}, J=4.8,2.8 \mathrm{~Hz}, 1 \mathrm{H}$, CHCHS), 7.48 (d, $J=4.8 \mathrm{~Hz}, 1 \mathrm{H}, \mathrm{CHCHS}), 7.67(\mathrm{~d}, J=2.8 \mathrm{~Hz}$, $1 \mathrm{H}, \mathrm{CCHS}), 7.75$ (s, $\left.1 \mathrm{H}, \mathrm{CH}_{\text {triaz }}\right) \mathrm{ppm} .{ }^{13} \mathrm{C} \mathrm{NMR}(100 \mathrm{MHz}$, $\left.\mathrm{CDCl}_{3}\right): \delta=21.5,23.0,24.0,25.1,26.5,27.1,28.6,35.3,38.3,39.5$, 41.6, 46.6 (br., CB), 51.2, 78.8, 87.4, 119.5, 120.8, 126.0, 126.1, 132.4, $143.7 \mathrm{ppm}$. HRMS (ESI-TOF) $\mathrm{m} / \mathrm{z}$ : calcd. for $\mathrm{C}_{21} \mathrm{H}_{31} \mathrm{BN}_{3} \mathrm{O}_{2} \mathrm{~S}[\mathrm{M}+\mathrm{H}]^{+} 400.2228$; found 400.2225 .

(+)-Pinanediyl I(1R)-3-Methyl-1-(4-phenoxymethyl-3-yl-1,2,3-triazol-1-yl)butyl|boronate (8e): Yellow viscous oil (reaction time $8 \mathrm{~h}$, $97 \%) \cdot[\alpha]_{\mathrm{D}}^{20}=+17.0\left(c=1.3, \mathrm{CHCl}_{3}\right) \cdot{ }^{1} \mathrm{H} \mathrm{NMR}\left(400 \mathrm{MHz}, \mathrm{CDCl}_{3}\right)$ : $\delta=0.83\left(\mathrm{~s}, 3 \mathrm{H}\right.$, pinanyl $\left.\mathrm{CH}_{3}\right), 0.85\left[\mathrm{~d}, J=6.6 \mathrm{~Hz}, 3 \mathrm{H}, \mathrm{CH}\left(\mathrm{CH}_{3}\right)\right.$ 2], $0.94\left[\mathrm{~d}, J=6.5 \mathrm{~Hz}, 3 \mathrm{H}, \mathrm{CH}\left(\mathrm{CH}_{3}\right)_{2}\right], 1.07(\mathrm{~d}, J=11.0 \mathrm{~Hz}, 1 \mathrm{H}$, pinanyl $\left.H_{\text {endo }}\right), 1.28\left(\mathrm{~s}, 3 \mathrm{H}\right.$, pinanyl $\left.\mathrm{CH}_{3}\right), 1.30-1.36[\mathrm{~m}, 1 \mathrm{H}$, $\mathrm{CH}\left(\mathrm{CH}_{3}\right)_{2}$ ], $1.40\left(\mathrm{~s}, 3 \mathrm{H}\right.$, pinanyl $\left.\mathrm{CH}_{3}\right), 1.70-2.36(\mathrm{~m}, 7 \mathrm{H}$, pinanyl protons, BCHCH 2$), 4.34(\mathrm{dd}, J=8.7,1.8 \mathrm{~Hz}, 1 \mathrm{H}, \mathrm{CHOB}), 4.55$ $(\mathrm{dd}, J=10.2,5.8 \mathrm{~Hz}, 1 \mathrm{H}, \mathrm{BCH}), 5.21\left(\mathrm{~s}, 2 \mathrm{H}, \mathrm{OCH}_{2}\right), 6.96(\mathrm{t}, J$ $\left.=7.4 \mathrm{~Hz}, 1 \mathrm{H}, \mathrm{H}_{\text {arom }}\right), 6.99\left(\mathrm{~d}, J=7.9 \mathrm{~Hz}, 2 \mathrm{H}, \mathrm{H}_{\mathrm{arom}}\right), 7.28(\mathrm{t}, J$ $\left.=7.6 \mathrm{~Hz}, 2 \mathrm{H}, \mathrm{H}_{\text {arom }}\right), 7.69\left(\mathrm{~s}, 1 \mathrm{H}, \mathrm{C} H_{\text {triaz }}\right) \mathrm{ppm} .{ }^{13} \mathrm{C} \mathrm{NMR}$ $\left(100 \mathrm{MHz} \mathrm{CDCl}_{3}\right): \delta=21.6,22.9,24.0,25.1,26.5,27.1,28.5,35.2$, 38.3, 39.4, 41.5, 46.8 (br., CB), 51.2, 62.4, 78.8, 87.4, 115.0, 121.2, 122.8, 129.5, 144.0, $158.5 \mathrm{ppm}$. HRMS (ESI-TOF) $\mathrm{m} / \mathrm{z}$ : calcd. for $\mathrm{C}_{24} \mathrm{H}_{35} \mathrm{BN}_{3} \mathrm{O}_{3}[\mathrm{M}+\mathrm{H}]^{+} 424.2770$; found 424.2788 .

[(1R)-1-(4-Ethoxycarbonyl-1,2,3-triazol-1-yl)-3-methylbutyl]boronic Acid (9a): Following the general procedure reported above, pinanediol removal from $\mathbf{8 a}$ by transesterification reaction afforded $\mathbf{9 a}$ as a white solid $(76 \%$ yield $)$, m.p. $115-117^{\circ} \mathrm{C}$ dec. $[a]_{\mathrm{D}}^{20}=+9.2(c=$ $\left.0.8, \mathrm{CH}_{3} \mathrm{OH}\right) .{ }^{1} \mathrm{H}$ NMR $\left(400 \mathrm{MHz}, \mathrm{CD}_{3} \mathrm{OD}\right): \delta=0.84[\mathrm{~d}, J=$ $\left.6.5 \mathrm{~Hz}, 3 \mathrm{H}, \mathrm{CH}\left(\mathrm{CH}_{3}\right)_{2}\right], 0.92\left[\mathrm{~d}, J=6.4 \mathrm{~Hz}, 3 \mathrm{H}, \mathrm{CH}\left(\mathrm{CH}_{3}\right)_{2}\right]$, 1.14-1.24 [m, $\left.1 \mathrm{H}, \mathrm{CH}\left(\mathrm{CH}_{3}\right)_{2}\right], 1.37(\mathrm{t}, J=6.8 \mathrm{~Hz}, 3 \mathrm{H}$, $\left.\mathrm{OCH}_{2} \mathrm{CH}_{3}\right), 1.70-1.76\left(\mathrm{~m}, 1 \mathrm{H}, \mathrm{BCHCH}_{2}\right), 1.90-1.97(\mathrm{~m}, 1 \mathrm{H}$, $\left.\mathrm{BCHCH}_{2}\right), 4.38\left(\mathrm{q}, J=6.8 \mathrm{~Hz}, 2 \mathrm{H}, \mathrm{OCH}_{2} \mathrm{CH}_{3}\right), 4.53(\mathrm{dd}, J=$ 10.8, $3.8 \mathrm{~Hz}, 1 \mathrm{H}, \mathrm{BCH}), 8.54\left(\mathrm{~s}, 1 \mathrm{H}, \mathrm{CH}_{\text {triaz }}\right) \mathrm{ppm} .{ }^{13} \mathrm{C} \mathrm{NMR}$ (100 MHz, $\left.\mathrm{CD}_{3} \mathrm{OD}\right): \delta=14.5,21.4,23.3,26.2,41.7,51.3$ (br., $C \mathrm{~B}$ ), $62.1,129.4,140.5,162.2 \mathrm{ppm}$. HRMS (ESI-TOF) $\mathrm{m} / \mathrm{z}$ : calcd. for $\mathrm{C}_{10} \mathrm{H}_{19} \mathrm{BN}_{3} \mathrm{O}_{4}[\mathrm{M}+\mathrm{H}]^{+}$256.1465; found 256.1468.

[(1R)-1-(4-Carboxy-1,2,3-triazol-1-yl)-3-methylbutyl]boronic Acid (9b): White solid (95\%), m.p. $123-127^{\circ} \mathrm{C} \mathrm{dec} .[\alpha]_{\mathrm{D}}^{20}=+4.5(c=0.4$, $\mathrm{CH}_{3} \mathrm{OH}$ ). ${ }^{1} \mathrm{H} \mathrm{NMR}\left(400 \mathrm{MHz}, \mathrm{CD}_{3} \mathrm{OD}\right): \delta=0.86[\mathrm{~d}, J=6.6 \mathrm{~Hz}$, $\left.3 \mathrm{H}, \mathrm{CH}\left(\mathrm{CH}_{3}\right)_{2}\right], 0.94\left[\mathrm{~d}, J=6.5 \mathrm{~Hz}, 3 \mathrm{H}, \mathrm{CH}\left(\mathrm{CH}_{3}\right)_{2}\right], 1.20-1.27$ $\left[\mathrm{m}, 1 \mathrm{H}, \mathrm{CH}\left(\mathrm{CH}_{3}\right)_{2}\right], 1.72$ (ddd, $J=14.3,8.9,4.5 \mathrm{~Hz}, 1 \mathrm{H}$, $\mathrm{BCHCH}_{2}$ ), 1.94 (ddd, $\left.J=14.3,11.2,4.7 \mathrm{~Hz}, 1 \mathrm{H}, \mathrm{BCHCH}_{2}\right), 4.58$ $(\mathrm{dd}, J=11.2,4.5 \mathrm{~Hz}, 1 \mathrm{H}, \mathrm{BC} H), 8.51\left(\mathrm{~s}, 1 \mathrm{H}, \mathrm{C} H_{\text {triaz }}\right) \mathrm{ppm} .{ }^{13} \mathrm{C}$ NMR (100 MHz, $\left.\mathrm{CD}_{3} \mathrm{OD}\right): \delta=21.4,23.3,26.3,41.7,51.1$ (br.,
461 
CB), 129.8, 141.0, $163.1 \mathrm{ppm}$. HRMS (ESI-TOF) $\mathrm{m} / \mathrm{z}$ : calcd. for $\mathrm{C}_{8} \mathrm{H}_{15} \mathrm{BN}_{3} \mathrm{O}_{4}[\mathrm{M}+\mathrm{H}]^{+}$228.1152; found 228.1148

[(1R)-3-Methyl-1-(4-phenyl-1,2,3-triazol-1-yl)butyl|boronic Acid (9c): Cream-colored solid $(80 \%)$, m.p. $127-132{ }^{\circ} \mathrm{C}$ dec. $[\alpha]_{\mathrm{D}}^{20}=+8.0$ $\left(c=0.5, \mathrm{CH}_{3} \mathrm{OH}\right) .{ }^{1} \mathrm{H}$ NMR $\left(400 \mathrm{MHz}, \mathrm{CD}_{3} \mathrm{OD}\right): \delta=0.92[\mathrm{~d}, J$ $\left.=6.6 \mathrm{~Hz}, 3 \mathrm{H}, \mathrm{CH}\left(\mathrm{CH}_{3}\right)_{2}\right], 0.98\left[\mathrm{~d}, J=6.5 \mathrm{~Hz}, 3 \mathrm{H}, \mathrm{CH}\left(\mathrm{CH}_{3}\right)_{2}\right]$, 1.31-1.38 [m, $\left.1 \mathrm{H}, \mathrm{CH}\left(\mathrm{CH}_{3}\right)_{2}\right], 1.84(\mathrm{ddd}, J=14.6,9.3,4.1 \mathrm{~Hz}, 1$ $\mathrm{H}, \mathrm{BCHCH} \mathrm{H}_{2}$ ), 2.14 (ddd, $J=14.6,11.5,4.5 \mathrm{~Hz}, 1 \mathrm{H}, \mathrm{BCHCH}_{2}$ ), $4.69(\mathrm{dd}, J=11.5,4.1 \mathrm{~Hz}, 1 \mathrm{H}, \mathrm{BCH}), 7.51-7.58\left(\mathrm{~m}, 3 \mathrm{H}, \mathrm{H}_{\text {arom }}\right)$, $7.86\left(\mathrm{~d}, J=6.8 \mathrm{~Hz}, 2 \mathrm{H}, \mathrm{H}_{\text {arom }}\right), 8.94\left(\mathrm{~s}, 1 \mathrm{H}, \mathrm{C} H_{\text {triaz }}\right) \mathrm{ppm} .{ }^{13} \mathrm{C}$ NMR $\left(100 \mathrm{MHz}, \mathrm{CD}_{3} \mathrm{OD}\right): \delta=21.3,23.3,26.3,40.8,54.6$ (br., CB), 125.7, 126.1, 127.6, 130.7, 131.9, 145.0 ppm. HRMS (ESITOF) $\mathrm{m} / \mathrm{z}$ : calcd. for $\mathrm{C}_{13} \mathrm{H}_{19} \mathrm{BN}_{3} \mathrm{O}_{2}[\mathrm{M}+\mathrm{H}]^{+}$260.1567; found 260.1563

531 [(1R)-3-Methyl-1-(4-thiophen-3-yl-1,2,3-triazol-1-yl)butyl|boronic Acid (9d): Cream-colored solid (92\%), m.p. $130-132{ }^{\circ} \mathrm{C}$ dec. $[\alpha]_{\mathrm{D}}^{20}$ $=+6.2\left(c=0.6, \mathrm{CH}_{3} \mathrm{OH}\right) .{ }^{1} \mathrm{H}$ NMR $\left(400 \mathrm{MHz}, \mathrm{CD}_{3} \mathrm{OD}\right): \delta=0.91$ $\left[\mathrm{d}, J=6.6 \mathrm{~Hz}, 3 \mathrm{H}, \mathrm{CH}\left(\mathrm{CH}_{3}\right)_{2}\right], 0.98\left[\mathrm{~d}, J=6.6 \mathrm{~Hz}, 3 \mathrm{H}, \mathrm{CH}\left(\mathrm{CH}_{3}\right)\right.$ 2], $1.26-1.38\left[\mathrm{~m}, 1 \mathrm{H}, \mathrm{CH}\left(\mathrm{CH}_{3}\right)_{2}\right.$ ], 1.82 (ddd, $J=14.6,9.3,4.3 \mathrm{~Hz}$,

$\left.5361 \mathrm{H}, \mathrm{BCHCH} \mathrm{H}_{2}\right), 2.10$ (ddd, $J=14.6,11.5,4.6 \mathrm{~Hz}, 1 \mathrm{H}, \mathrm{BCHCH}_{2}$ ), $4.66(\mathrm{dd}, J=11.5,4.3 \mathrm{~Hz}, 1 \mathrm{H}, \mathrm{BCH}), 7.56(\mathrm{~d}, J=4.9 \mathrm{~Hz}, 1 \mathrm{H}$, CHCHS), 7.66 (dd, $J=5.1,2.8 \mathrm{~Hz}, 1 \mathrm{H}, \mathrm{CHCHS}), 8.04(\mathrm{~d}, J=$ $2.0 \mathrm{~Hz}, 1 \mathrm{H}, \mathrm{CC} H \mathrm{~S}), 8.78\left(\mathrm{~s}, 1 \mathrm{H}, \mathrm{C} H_{\text {triaz }}\right) \mathrm{ppm} .{ }^{13} \mathrm{C} \mathrm{NMR}$ (100 MHz, $\left.\mathrm{CD}_{3} \mathrm{OD}\right): \delta=21.3,23.3,26.3,40.9,54.3$ (br., $C \mathrm{~B}$ ),

$541125.1,126.3,126.5,127.3,129.3,141.3 \mathrm{ppm}$. HRMS (ESI-TOF) $\mathrm{m} / \mathrm{z}$ : calcd. for $\mathrm{C}_{11} \mathrm{H}_{17} \mathrm{BN}_{3} \mathrm{O}_{2} \mathrm{~S}[\mathrm{M}+\mathrm{H}]^{+}$266.1131; found 266.1137 .

[(1R)-3-Methyl-1-(4-phenoxymethyl-3-yl-1,2,3-triazol-1-yl)butyl]boronic Acid (9e): White solid (77\%), m.p. $118-123{ }^{\circ} \mathrm{C}$ dec. $[\alpha]_{\mathrm{D}}^{20}=$ $+2.9\left(c=1.2, \mathrm{CH}_{3} \mathrm{OH}\right) .{ }^{1} \mathrm{H}$ NMR $\left(400 \mathrm{MHz}, \mathrm{CD}_{3} \mathrm{OD}\right): \delta=0.87$

$546\left[\mathrm{~d}, J=6.6 \mathrm{~Hz}, 3 \mathrm{H}, \mathrm{CH}\left(\mathrm{CH}_{3}\right)_{2}\right], 0.95\left[\mathrm{~d}, J=6.5 \mathrm{~Hz}, 3 \mathrm{H}, \mathrm{CH}\left(\mathrm{CH}_{3}\right)\right.$ 2], $1.20-1.29\left[\mathrm{~m}, 1 \mathrm{H}, \mathrm{CH}\left(\mathrm{CH}_{3}\right)_{2}\right], 1.73$ (ddd, $J=14.5,9.1,4.5 \mathrm{~Hz}$, $1 \mathrm{H}, \mathrm{BCHCH} \mathrm{H}_{2}$ ) 1.97 (ddd, $J=14.5,11.3,4.6 \mathrm{~Hz}, 1 \mathrm{H}, \mathrm{BCHCH}_{2}$ ), $4.64(\mathrm{dd}, J=11.3,4.5 \mathrm{~Hz}, 1 \mathrm{H}, \mathrm{BCH}), 5.24\left(\mathrm{~s}, 2 \mathrm{H}, \mathrm{OCH}_{2}\right), 6.98$ (tt, $\left.J=7.4,1.0 \mathrm{~Hz}, 1 \mathrm{H}, \mathrm{H}_{\text {arom }}\right), 7.02(\mathrm{~d}, J=8.7,1.0 \mathrm{~Hz}, 2 \mathrm{H}$,

$\left.551 \mathrm{H}_{\text {arom }}\right), 7.30\left(\mathrm{dd}, J=8.7,7.4 \mathrm{~Hz}, 2 \mathrm{H}, \mathrm{H}_{\text {arom }}\right), 8.30(\mathrm{~s}, 1 \mathrm{H}$, $\left.\mathrm{CH}_{\text {triaz }}\right)$ ppm. $\left.{ }^{13} \mathrm{C} \mathrm{NMR} \mathrm{(100} \mathrm{MHz,} \mathrm{CD}_{3} \mathrm{OD}\right): \delta=21.4,23.3,26.3$, 41.4, 51.9 (br., CB), 61.4, 116.1, 122.7, 126.6, 130.6, 143.3, $159.3 \mathrm{ppm}$. HRMS (ESI-TOF) $\mathrm{m} / \mathrm{z}$ : calcd. for $\mathrm{C}_{14} \mathrm{H}_{21} \mathrm{BN}_{3} \mathrm{O}_{3}[\mathrm{M}+$ $\mathrm{H}]^{+}$290.1673; found 290.1673 .

556 (+)-Pinanediyl [(1 R)-1-Azido-2-phenylethyl]boronate (16): Starting from $14^{[17]}$ and following the procedure described for the synthesis of 7, compound 16 was recovered as a yellowish oil $(94 \%)$. $[\alpha]_{D}^{20}=$ $+8.7\left(c=1.0, \mathrm{CHCl}_{3}\right) .{ }^{1} \mathrm{H}$ NMR $\left(400 \mathrm{MHz}, \mathrm{CDCl}_{3}\right): \delta=0.83(\mathrm{~s}$, $3 \mathrm{H}$, pinanyl $\left.\mathrm{CH}_{3}\right), 0.98\left(\mathrm{~d}, J=11.0 \mathrm{~Hz}, 1 \mathrm{H}\right.$, pinanyl $\left.H_{\text {endo }}\right), 1.29$

561 (s, $3 \mathrm{H}$, pinanyl $\left.\mathrm{CH}_{3}\right), 1.38\left(\mathrm{~s}, 3 \mathrm{H}\right.$, pinanyl $\left.\mathrm{CH}_{3}\right), 1.85-2.37(\mathrm{~m}, 5$ $\mathrm{H}$, pinanyl protons), 2.95 (dd, $J=14.0,9.0 \mathrm{~Hz}, 1 \mathrm{H}, \mathrm{BCHCH}_{2}$ ), $3.03\left(\mathrm{dd}, J=14.0,5.7 \mathrm{~Hz}, 1 \mathrm{H}, \mathrm{BCHCH}_{2}\right), 3.38(\mathrm{dd}, J=9.0$, $5.7 \mathrm{~Hz}, 1 \mathrm{H}, \mathrm{BCH}), 4.33(\mathrm{dd}, J=8.7,1.7 \mathrm{~Hz}, 1 \mathrm{H}, \mathrm{CHOB}), 7.19$ $7.32\left(\mathrm{~m}, 5 \mathrm{H}, \mathrm{H}_{\text {arom }}\right)$ ppm. ${ }^{13} \mathrm{C}$ NMR $\left(100 \mathrm{MHz}, \mathrm{CDCl}_{3}\right): \delta=24.1$, 26.5, 27.1, 28.6, 35.3, 36.9, 38.3, 39.5, 49.8 (br., CB), 51.2, 78.7, 87.1, 126.8, 128.6, 129.3, 138.8 ppm. $\mathrm{C}_{18} \mathrm{H}_{24} \mathrm{BN}_{3} \mathrm{O}_{2}$ (325.22): calcd. C 66.48, H 7.44, N 12.92; found C 66.25, H 7.68, N 12.72 .

(+)-Pinanediyl [(1R)-1-Azido-2-(3-tert-butoxycarbonylphenyl)ethyl]boronate (17): Starting from $15^{[17]}$ and following the procedure de-

571 scribed for the synthesis of $\mathbf{7}$, compound $\mathbf{1 7}$ was recovered as a yellowish oil $(97 \%) \cdot[\alpha]_{\mathrm{D}}^{20}=+11.9\left(c=1.8, \mathrm{CHCl}_{3}\right) .{ }^{1} \mathrm{H}$ NMR $\left(400 \mathrm{MHz}, \mathrm{CDCl}_{3}\right): \delta=0.82\left(\mathrm{~s}, 3 \mathrm{H}\right.$, pinanyl $\left.\mathrm{CH}_{3}\right), 0.93(\mathrm{~d}, J=$ $11.0 \mathrm{~Hz}, 1 \mathrm{H}$, pinanyl $\left.H_{\text {endo }}\right), 1.27\left(\mathrm{~s}, 3 \mathrm{H}\right.$, pinanyl $\left.\mathrm{CH}_{3}\right), 1.37$ (s, 3 $\mathrm{H}$, pinanyl $\left.\mathrm{CH}_{3}\right), 1.59(\mathrm{~s}, 9 \mathrm{H}, t \mathrm{Bu}), 1.86-2.38(\mathrm{~m}, 5 \mathrm{H}$, pinanyl protons), 2.99 (dd, $J=13.9,8.5 \mathrm{~Hz}, 1 \mathrm{H}, \mathrm{BCHCH}_{2}$ ), 3.05 (dd, $J=$ $\left.13.9,6.0 \mathrm{~Hz}, 1 \mathrm{H}, \mathrm{BCHCH}_{2}\right), 3.39(\mathrm{dd}, J=8.5,6.0 \mathrm{~Hz}, 1 \mathrm{H}, \mathrm{BCH})$, $4.34(\mathrm{~d}, J=7.6 \mathrm{~Hz}, 1 \mathrm{H}, \mathrm{CHOB}), 7.34\left(\mathrm{t}, J=7.7 \mathrm{~Hz}, 1 \mathrm{H}, \mathrm{H}_{\text {arom }}\right)$, $7.44\left(\mathrm{~d}, J=7.7 \mathrm{~Hz}, 1 \mathrm{H}, \mathrm{H}_{\text {arom }}\right), 7.86\left(\mathrm{~d}, J=7.7 \mathrm{~Hz}, 1 \mathrm{H}, \mathrm{H}_{\text {arom }}\right)$, $7.89\left(\mathrm{~s}, 1 \mathrm{H}, \mathrm{H}_{\text {arom }}\right) \mathrm{ppm} .{ }^{13} \mathrm{C} \mathrm{NMR}\left(100 \mathrm{MHz}, \mathrm{CDCl}_{3}\right): \delta=24.0$, 26.4, 27.1, 28.3, 28.5, 35.2, 36.7, 38.2, 39.5, 49.5 (br., CB), 51.1, 78.7, 81.0, 87.1, 128.0, 128.4, 130.2, 132.3, 133.4, 138.8, 165.7 ppm. $\mathrm{C}_{23} \mathrm{H}_{32} \mathrm{BN}_{3} \mathrm{O}_{4}$ (425.33): calcd. $\mathrm{C} 64.95, \mathrm{H}$ 7.58, N 9.88; found $\mathrm{C}$ 65.18, H 7.81, N 9.64.

[(1 R)-1-(4-Ethoxycarbonyl-1,2,3-triazol-1-yl)-2-phenylethyl]boronic Acid (18a): According to the general procedure reported above, CuAAC reaction between azido boronate $\mathbf{1 6}$ and ethyl propiolate (reaction time $2 \mathrm{~h}$ ) followed by deprotection of pinanediol boronate ester through transesterification reaction afforded 18a as a white solid $(75 \%$ overall yield $)$, m.p. $147-149^{\circ} \mathrm{C}$ dec. $[\alpha]_{\mathrm{D}}^{20}=-53.7(c=$ $\left.1.1, \mathrm{CH}_{3} \mathrm{OH}\right) .{ }^{1} \mathrm{H}$ NMR $\left(400 \mathrm{MHz}, \mathrm{CD}_{3} \mathrm{OD}\right): \delta=1.34(\mathrm{t}, J=$ $\left.7.1 \mathrm{~Hz}, 3 \mathrm{H}, \mathrm{OCH}_{2} \mathrm{CH}_{3}\right), 3.20(\mathrm{dd}, J=14.0,9.9 \mathrm{~Hz}, 1 \mathrm{H}$, $\left.\mathrm{BCHCH}_{2}\right), 3.25-3.31\left(\mathrm{~m}, 1 \mathrm{H}, \mathrm{BCHCH}{ }_{2}\right.$ and $\left.\mathrm{CD}_{3} \mathrm{OD}\right), 4.33$ (q, $J$ $\left.=7.1 \mathrm{~Hz}, 2 \mathrm{H}, \mathrm{OCH}_{2} \mathrm{CH}_{3}\right), 4.62(\mathrm{dd}, J=9.9,4.9 \mathrm{~Hz}, 1 \mathrm{H}, \mathrm{BCH})$, $6.97\left(\mathrm{~d}, J=7.0 \mathrm{~Hz}, 2 \mathrm{H}, \mathrm{H}_{\text {arom }}\right), 7.14-7.20\left(\mathrm{~m}, 3 \mathrm{H}, \mathrm{H}_{\text {arom }}\right), 8.16$ $\left(\mathrm{s}, 1 \mathrm{H}, \mathrm{CH}_{\text {triaz }}\right) \mathrm{ppm} .{ }^{13} \mathrm{C} \mathrm{NMR}\left(100 \mathrm{MHz}, \mathrm{CD}_{3} \mathrm{OD}\right): \delta=14.5$, 39.2, 55.4 (br., CB), 62.2, 127.7, 129.5, 129.7, 130.4, 139.3, $162.3 \mathrm{ppm}, \mathrm{C}-4$ triazole ring not seen. HRMS (ESI-TOF) $\mathrm{m} / \mathrm{z}$ : calcd. for $\mathrm{C}_{13} \mathrm{H}_{17} \mathrm{BN}_{3} \mathrm{O}_{4}[\mathrm{M}+\mathrm{H}]^{+}$290.1309; found 290.1295 .

[(1R)-1-(4-Carboxy-1,2,3-triazol-1-yl)-2-phenylethyl]boronic Acid (18b): White solid (reaction time 2 h, $85 \%$ overall yield), m.p. 120 $122{ }^{\circ} \mathrm{C}$ dec. $[\alpha]_{\mathrm{D}}^{20}=-43.4\left(c=1.0, \mathrm{CH}_{3} \mathrm{OH}\right) .{ }^{1} \mathrm{H}$ NMR $(400 \mathrm{MHz}$, $\left.\mathrm{CD}_{3} \mathrm{OD}\right): \delta=3.18\left(\mathrm{dd}, J=14.0,10.0 \mathrm{~Hz}, 1 \mathrm{H}, \mathrm{BCHCH}_{2}\right), 3.27$ $3.32\left(\mathrm{~m}, 1 \mathrm{H}, \mathrm{BCHCH} \mathrm{H}_{2}\right.$ and $\left.\mathrm{CD}_{3} \mathrm{OD}\right), 4.68(\mathrm{dd}, J=10.0,5.6 \mathrm{~Hz}$, $1 \mathrm{H}, \mathrm{BCH}), 7.00\left(\mathrm{~d}, J=6.6 \mathrm{~Hz}, 2 \mathrm{H}, \mathrm{H}_{\text {arom }}\right), 7.14-7.22(\mathrm{~m}, 3 \mathrm{H}$, $\left.\mathrm{H}_{\text {arom }}\right), 8.15$ (s, $\left.1 \mathrm{H}, \mathrm{CH}_{\text {triaz }}\right)$ ppm. ${ }^{13} \mathrm{C}$ NMR $\left(100 \mathrm{MHz}, \mathrm{CD}_{3} \mathrm{OD}\right)$ : $\delta=39.3,54.5$ (br., CB), 127.8, 129.5, 129.8, 130.3, 139.2, 140.4, $163.2 \mathrm{ppm}$. HRMS (ESI-TOF) $\mathrm{m} / \mathrm{z}$ : calcd. for $\mathrm{C}_{11} \mathrm{H}_{13} \mathrm{BN}_{3} \mathrm{O}_{4}[\mathrm{M}+$ $\mathrm{H}]^{+}$262.0996; found 262.0994 .

[(1R)-1-(4-Phenyl-1,2,3-triazol-1-yl)-2-phenylethyl|boronic Acid (18c): White solid (reaction time $16 \mathrm{~h}, 70 \%$ overall yield), m.p. $151-$ $153{ }^{\circ} \mathrm{C}$ dec. $[\alpha]_{\mathrm{D}}^{20}=-62.3\left(c=1.3, \mathrm{CH}_{3} \mathrm{OH}\right) .{ }^{1} \mathrm{H}$ NMR $(400 \mathrm{MHz}$, $\left.\mathrm{CD}_{3} \mathrm{OD}\right): \delta=3.25-3.31\left(\mathrm{~m}, 1 \mathrm{H}, \mathrm{BCHCH}_{2}\right.$ and $\left.\mathrm{CD}_{3} \mathrm{OD}\right), 3.39(\mathrm{dd}$, $\left.J=14.1,5.7 \mathrm{~Hz}, 1 \mathrm{H}, \mathrm{BCHCH}_{2}\right), 4.84(\mathrm{dd}, J=10.0,5.7 \mathrm{~Hz}, 1 \mathrm{H}$, $\mathrm{BCH}), 7.12\left(\mathrm{~d}, J=6.9 \mathrm{~Hz}, 2 \mathrm{H}, \mathrm{H}_{\text {arom }}\right), 7.16-7.25\left(\mathrm{~m}, 3 \mathrm{H}, \mathrm{H}_{\text {arom }}\right)$, 7.44-7.52 (m, $\left.3 \mathrm{H}, \mathrm{H}_{\text {arom }}\right), 7.73\left(\mathrm{~d}, J=6.7 \mathrm{~Hz}, 2 \mathrm{H}, \mathrm{H}_{\text {arom }}\right), 8.51$ (s, $\left.1 \mathrm{H}, \mathrm{CH}_{\text {triaz }}\right)$ ppm. ${ }^{13} \mathrm{C}$ NMR $\left(100 \mathrm{MHz}, \mathrm{CD}_{3} \mathrm{OD}\right): \delta=38.8$, 56.3 (br., $C \mathrm{~B}$ ), 125.1, 127.2, 127.8, 128.1, 129.7, 129.9, 130.4, 131.1, 138.8, 145.9 ppm. HRMS (ESI-TOF) $m / z$ : calcd. for $\mathrm{C}_{16} \mathrm{H}_{17} \mathrm{BN}_{3} \mathrm{O}_{2}$ $[\mathrm{M}+\mathrm{H}]^{+}$294.1411; found 294.1423.

I(1R)-2-Phenyl-1-(4-thiophen-3-yl-1,2,3-triazol-1-yl)ethyl|boronic Acid (18d): Cream-colored solid (reaction time $16 \mathrm{~h}, 80 \%$ overall yield), m.p. $141-143{ }^{\circ} \mathrm{C}$ dec. $[\alpha]_{\mathrm{D}}^{20}=-67.3\left(c=1.1, \mathrm{CH}_{3} \mathrm{OH}\right) .{ }^{1} \mathrm{H}$ NMR $\left(400 \mathrm{MHz}, \mathrm{CD}_{3} \mathrm{OD}\right): \delta=3.25-3.32\left(\mathrm{~m}, 1 \mathrm{H}, \mathrm{BCHCH}_{2}\right.$ and $\mathrm{CD}_{3} \mathrm{OD}$ ), 3.40 (dd, $J=14.2,5.7 \mathrm{~Hz}, 1 \mathrm{H}, \mathrm{BCHCH}_{2}$ ), 4.87 (dd, $J$ $=10.6,5.7 \mathrm{~Hz}, 1 \mathrm{H}, \mathrm{BCH}), 7.13\left(\mathrm{~d}, J=6.9 \mathrm{~Hz}, 2 \mathrm{H}, \mathrm{H}_{\text {arom }}\right), 7.17-$ $7.25\left(\mathrm{~m}, 3 \mathrm{H}, \mathrm{H}_{\text {arom }}\right), 7.47\left(\mathrm{~d}, J=5.0 \mathrm{~Hz}, 1 \mathrm{H}, \mathrm{H}_{\text {arom }}\right), 7.63(\mathrm{dd}, J$ $\left.=5.0,2.8 \mathrm{~Hz}, 1 \mathrm{H}, \mathrm{H}_{\text {arom }}\right), 7.94\left(\mathrm{~d}, J=1.9 \mathrm{~Hz}, 1 \mathrm{H}, \mathrm{H}_{\text {arom }}\right), 8.57$ (s, $\left.1 \mathrm{H}, \mathrm{CH}_{\text {triaz }}\right)$ ppm. ${ }^{13} \mathrm{C}$ NMR $\left(100 \mathrm{MHz}, \mathrm{CD}_{3} \mathrm{OD}\right): \delta=38.7$, 56.7 (br., CB), 125.3, 126.0, 126.5, 127.5, 128.1, 129.2, 129.7, 129.8, $138.6,141.5 \mathrm{ppm}$. HRMS (ESI-TOF) $\mathrm{m} / \mathrm{z}$ : calcd. for $\mathrm{C}_{14} \mathrm{H}_{15} \mathrm{BN}_{3} \mathrm{O}_{2} \mathrm{~S}[\mathrm{M}+\mathrm{H}]^{+} 300.0975$; found 300.0987 .

[(1R)-2-Phenyl-1-(4-phenoxymethyl-1,2,3-triazol-1-yl)ethyl|boronic Acid (18e): White solid (reaction time $16 \mathrm{~h}, 79 \%$ overall yield), m.p. $113-115^{\circ} \mathrm{C}$ dec. $[\alpha]_{\mathrm{D}}^{20}=-50.1\left(c=0.8, \mathrm{CH}_{3} \mathrm{OH}\right) .{ }^{1} \mathrm{H} \mathrm{NMR}$ $\left(400 \mathrm{MHz}, \mathrm{CD}_{3} \mathrm{OD}\right): \delta=3.13(\mathrm{dd}, J=14.0,9.8 \mathrm{~Hz}, 1 \mathrm{H}$ $\left.\mathrm{BCHCH}_{2}\right), 3.24-3.32\left(\mathrm{~m}, 1 \mathrm{H}, \mathrm{BCHCH}_{2}\right.$ and $\left.\mathrm{CD}_{3} \mathrm{OD}\right), 4.67(\mathrm{dd}$, $J=9.8,5.8 \mathrm{~Hz}, 1 \mathrm{H}, \mathrm{BCH}), 5.10\left(\mathrm{~s}, 2 \mathrm{H}, \mathrm{OCH}_{2}\right), 6.93-6.97(\mathrm{~m}, 5$ $\left.\mathrm{H}, \mathrm{H}_{\text {arom }}\right), 7.14-7.16$ (m, $\left.3 \mathrm{H}, \mathrm{H}_{\text {arom }}\right), 7.25-7.29$ (m, $\left.2 \mathrm{H}, \mathrm{H}_{\text {arom }}\right)$, $7.92\left(\mathrm{~s}, 1 \mathrm{H}, \mathrm{C} H_{\text {triaz }}\right)$ ppm. ${ }^{13} \mathrm{C}$ NMR $\left(100 \mathrm{MHz}, \mathrm{CD}_{3} \mathrm{OD}\right): \delta=$
581 
39.4, 53.9 (br., CB), 62.1, 116.0, 122.3, 127.7, 129.5, 129.76, 129.85, 130.5, 139.4, $159.6 \mathrm{ppm}, \mathrm{C}-4$ triazole ring not seen. HRMS (ESITOF) $m / z$ : calcd. for $\mathrm{C}_{17} \mathrm{H}_{19} \mathrm{BN}_{3} \mathrm{O}_{3}[\mathrm{M}+\mathrm{H}]^{+} 324.1517$; found 324.1513.

[(1R)-1-(4-Carboxy-1,2,3-triazol-1-yl)-2-(3-carboxyphenyl)ethyl] boronic Acid (19a): According to the general procedure reported above, the product of the $\mathrm{CuAAC}$ reaction between azido boronate 17 and ethyl propiolate (reaction time $2 \mathrm{~h}$ ) was firstly subjected to tert-butyl group removal (trifluoroacetic anhydride $25 \% \mathrm{v} / \mathrm{v}$ in $\mathrm{CH}_{2} \mathrm{Cl}_{2}, 2 \mathrm{~mL}$, room temp., $5 \mathrm{~h}$ ) followed by deprotection of pin-

651 anediol boronate ester through transesterification reaction afforded 19a as a white solid ( $64 \%$ overall yield), m.p. $157-159^{\circ} \mathrm{C}$ dec. $[a]$ ${ }_{\mathrm{D}}^{20}=-54.9\left(c=1.2, \mathrm{CH}_{3} \mathrm{OH}\right) .{ }^{1} \mathrm{H} \mathrm{NMR}\left(400 \mathrm{MHz}, \mathrm{CD}_{3} \mathrm{OD}\right): \delta=$ $1.35\left(\mathrm{t}, J=7.1 \mathrm{~Hz}, 3 \mathrm{H}, \mathrm{OCH}_{2} \mathrm{CH}_{3}\right), 3.24(\mathrm{dd}, J=14.0,10.0 \mathrm{~Hz}$, $\left.1 \mathrm{H}, \mathrm{BCHCH} \mathrm{H}_{2}\right), 3.34\left(\mathrm{dd}, J=14.0,5.2 \mathrm{~Hz}, 1 \mathrm{H}, \mathrm{BCHCH}_{2}\right), 4.34$

656 (q, $\left.J=7.1 \mathrm{~Hz}, 2 \mathrm{H}, \mathrm{OCH}_{2} \mathrm{CH}_{3}\right), 4.75(\mathrm{~m}, 1 \mathrm{H}, \mathrm{BCH}), 7.26(\mathrm{~d}, J=$ $\left.7.6 \mathrm{~Hz}, 1 \mathrm{H}, \mathrm{H}_{\text {arom }}\right), 7.33\left(\mathrm{t}, J=7.6 \mathrm{~Hz}, 1 \mathrm{H}, \mathrm{H}_{\text {arom }}\right), 7.69$ (s, $1 \mathrm{H}$, $\left.\mathrm{H}_{\text {arom }}\right), 7.85\left(\mathrm{~d}, J=7.6 \mathrm{~Hz}, 1 \mathrm{H}, \mathrm{H}_{\text {arom }}\right), 8.23\left(\mathrm{~s}, 1 \mathrm{H}, \mathrm{CH}_{\text {triaz }}\right) \mathrm{ppm}$. ${ }^{13} \mathrm{C}$ NMR (100 MHz, CD 3 OD): $\delta=14.5,39.1,54.4$ (br., $\left.C \mathrm{~B}\right), 62.1$, $128.5,129.2,129.6,130.3,131.2,134.5,139.9,162.1,169.4$ ppm, C-

6614 triazole ring not seen. HRMS (ESI-TOF) $\mathrm{m} / \mathrm{z}$ : calcd. for $\mathrm{C}_{14} \mathrm{H}_{17} \mathrm{BN}_{3} \mathrm{O}_{6}[\mathrm{M}+\mathrm{H}]^{+}$334.1204; found 334.1208.

I(1R)-2-(3-Carboxyphenyl)-1-(4-ethoxycarbonyl-1,2,3-triazol-1-yl)ethyl]boronic Acid (19b): White solid (reaction time 2 h, $55 \%$ overall yield), m.p. $108-110^{\circ} \mathrm{C}$ dec. $[\alpha]_{\mathrm{D}}^{20}=-49.2\left(c=1.5, \mathrm{CH}_{3} \mathrm{OH}\right) .{ }^{1} \mathrm{H}$ NMR (400 MHz, CD $\left.\mathrm{CD}_{3} \mathrm{OD}\right): \delta=3.26(\mathrm{dd}, J=14.0,10.1 \mathrm{~Hz}, 1 \mathrm{H}$, $\mathrm{BCHCH}_{2}$ ), 3.31-3.41 (m, $1 \mathrm{H}, \mathrm{BCHCH}_{2}$ and $\left.\mathrm{CD}_{3} \mathrm{OD}\right), 4.71$ (br., $1 \mathrm{H}, \mathrm{BCH}), 7.22\left(\mathrm{~d}, J=7.7 \mathrm{~Hz}, 1 \mathrm{H}, \mathrm{H}_{\mathrm{arom}}\right), 7.32(\mathrm{t}, J=7.7 \mathrm{~Hz}$, $\left.1 \mathrm{H}, \mathrm{H}_{\text {arom }}\right), 7.71\left(\mathrm{~s}, 1 \mathrm{H}, \mathrm{H}_{\text {arom }}\right), 7.84\left(\mathrm{~d}, J=7.7 \mathrm{~Hz}, 1 \mathrm{H}, \mathrm{H}_{\text {arom }}\right)$, 8.18 (s, $\left.1 \mathrm{H}, \mathrm{C} H_{\text {triaz }}\right)$ ppm. ${ }^{13} \mathrm{C} \mathrm{NMR}\left(100 \mathrm{MHz}, \mathrm{CD}_{3} \mathrm{OD}\right): \delta=$ $67139.1,53.9$ (br., CB), 128.5, 129.2, 129.6, 131.2, 132.2, 134.6, 139.9, $140.8,163.5,169.6 \mathrm{ppm}$. HRMS (ESI-TOF) $\mathrm{m} / \mathrm{z}$ : calcd. for $\mathrm{C}_{12} \mathrm{H}_{11} \mathrm{BN}_{3} \mathrm{O}_{6}[\mathrm{M}-\mathrm{H}]^{-}$304.0749; found 304.0739.

[(1R)-2-(3-Carboxyphenyl)-1-(4-phenyl-1,2,3-triazol-1-yl)ethyl]boronic Acid (19c): White solid (reaction time $16 \mathrm{~h}, 79 \%$ overall 676 yield), m.p. $88-90^{\circ} \mathrm{C}$ dec. $[a]_{\mathrm{D}}^{20}=-70.0\left(c=1.0, \mathrm{CH}_{3} \mathrm{OH}\right) \cdot{ }^{1} \mathrm{H}$ NMR (400 MHz, $\left.\mathrm{CD}_{3} \mathrm{OD}\right): \delta=3.26-3.32\left(\mathrm{~m}, 1 \mathrm{H}, \mathrm{BCHCH}_{2}\right.$ and $\left.\mathrm{CD}_{3} \mathrm{OD}\right), 3.39\left(\mathrm{dd}, J=14.1,5.8 \mathrm{~Hz}, 1 \mathrm{H}, \mathrm{BCHCH}_{2}\right), 4.74(\mathrm{dd}, J$ $=9.9,5.8 \mathrm{~Hz}, 1 \mathrm{H}, \mathrm{BCH}), 7.29-7.44\left(\mathrm{~m}, 5 \mathrm{H}, \mathrm{H}_{\text {arom }}\right), 7.71(\mathrm{~d}, J=$ $\left.7.4 \mathrm{~Hz}, 2 \mathrm{H}, \mathrm{H}_{\text {arom }}\right), 7.76\left(\mathrm{~s}, 1 \mathrm{H}, \mathrm{H}_{\text {arom }}\right), 7.85$ (d, J = 7.2 Hz, $1 \mathrm{H}$, $\left.681 \mathrm{H}_{\text {arom }}\right), 8.17\left(\mathrm{~s}, 1 \mathrm{H}, \mathrm{CH}_{\text {triaz }}\right) \mathrm{ppm} .{ }^{13} \mathrm{C} \mathrm{NMR}\left(100 \mathrm{MHz}, \mathrm{CD}_{3} \mathrm{OD}\right)$ : $\delta=39.1,54.0$ (br., CB), 123.6, 126.8, 129.2, 129.7, 130.00, 130.06, $131.2,132.2,134.7,140.0,147.1,169.6 \mathrm{ppm}, \mathrm{C}-4$ triazole ring not seen. HRMS (ESI-TOF) $\mathrm{m} / z$ : calcd. for $\mathrm{C}_{17} \mathrm{H}_{17} \mathrm{BN}_{3} \mathrm{O}_{4}[\mathrm{M}+\mathrm{H}]^{+}$ 338.1310 ; found 338.1310 .

686 I(1R)-2-(3-Carboxyphenyl)1-(4-thiophen-3-yl-1,2,3-triazol-1-yl)ethyl]boronic Acid (19d): Cream-colored solid (reaction time 16 h, 60\% overall yield), m.p. $185-187^{\circ} \mathrm{C}$ dec. $[\alpha]_{\mathrm{D}}^{20}=-69.4\left(c=0.7, \mathrm{CH}_{3} \mathrm{OH}\right)$. ${ }^{1} \mathrm{H}$ NMR (400 MHz, CD $\left.{ }_{3} \mathrm{OD}\right): \delta=3.32(\mathrm{dd}, J=14.2,10.2 \mathrm{~Hz}, 1$ $\left.\mathrm{H}, \mathrm{BCHCH}_{2}\right), 3.41$ (dd, $\left.J=14.2,5.6 \mathrm{~Hz}, 1 \mathrm{H}, \mathrm{BCHCH}_{2}\right), 4.76$ (dd,

$691 J=10.2,5.6 \mathrm{~Hz}, 1 \mathrm{H}, \mathrm{BCH}), 7.30-7.44\left(\mathrm{~m}, 3 \mathrm{H}, \mathrm{H}_{\text {arom }}\right), 7.54(\mathrm{dd}$, $\left.J=5.0,2.9 \mathrm{~Hz}, 1 \mathrm{H}, \mathrm{H}_{\text {arom }}\right), 7.75$ (s, $\left.1 \mathrm{H}, \mathrm{H}_{\text {arom }}\right), 7.79$ (d, $J=$ $\left.1.9 \mathrm{~Hz}, 1 \mathrm{H}, \mathrm{H}_{\text {arom }}\right), 7.84\left(\mathrm{~d}, J=6.8 \mathrm{~Hz}, 1 \mathrm{H}, \mathrm{H}_{\text {arom }}\right), 8.28$ (s, $1 \mathrm{H}$, $\mathrm{CH}_{\text {triaz }}$ ) ppm. $\left.{ }^{13} \mathrm{C} \mathrm{NMR} \mathrm{(100} \mathrm{MHz,} \mathrm{CD}_{3} \mathrm{OD}\right): \delta=38.8,55.2$ (br., CB), 124.1 (2 C), 126.6, 128.3, 129.3, 129.7, 131.1, 132.2, 134.5,

$696134.6,139.7,142.9,169.5 \mathrm{ppm}$. HRMS (ESI-TOF) $\mathrm{m} / \mathrm{z}$ : calcd. for $\mathrm{C}_{15} \mathrm{H}_{15} \mathrm{BN}_{3} \mathrm{O}_{4} \mathrm{~S}[\mathrm{M}+\mathrm{H}]^{+} 344.0874$; found 344.0873 .

[(1R)-2-(3-Carboxyphenyl)1-(4-phenoxymethyl-3-yl-1,2,3-triazol-1yl)ethyl|boronic Acid (19e): White solid (reaction time $8 \mathrm{~h}, 54 \%$ overall yield), m.p. $182-184{ }^{\circ} \mathrm{C}$ dec. $[\alpha]_{\mathrm{D}}^{20}=-37.1\left(c=1.3, \mathrm{CH}_{3} \mathrm{OH}\right)$.

$701{ }^{1} \mathrm{H}$ NMR (400 MHz, $\left.\mathrm{CD}_{3} \mathrm{OD}\right): \delta=3.21(\mathrm{dd}, J=14.1,10.1 \mathrm{~Hz}, 1$ $\left.\mathrm{H}, \mathrm{BCHCH}_{2}\right), 3.32\left(\mathrm{dd}, J=14.1,5.6 \mathrm{~Hz}, 1 \mathrm{H}, \mathrm{BCHCH}_{2}\right), 4.72$ (dd,
$J=10.1,5.6 \mathrm{~Hz}, 1 \mathrm{H}, \mathrm{BCH}), 5.10\left(\mathrm{~s}, 2 \mathrm{H}, \mathrm{OCH}_{2}\right), 6.92-6.96(\mathrm{~m}, 3$ $\left.\mathrm{H}, \mathrm{H}_{\text {arom }}\right), 7.17-7.28\left(\mathrm{~m}, 4 \mathrm{H}, \mathrm{H}_{\text {arom }}\right), 7.71\left(\mathrm{~s}, 1 \mathrm{H}, \mathrm{H}_{\text {arom }}\right), 7.82$ (d, $\left.J=7.7 \mathrm{~Hz}, 1 \mathrm{H}, \mathrm{H}_{\text {arom }}\right), 7.89$ (s, $\left.1 \mathrm{H}, \mathrm{CH}_{\text {triaz }}\right) \mathrm{ppm} .{ }^{13} \mathrm{C} \mathrm{NMR}$ (100 MHz, $\left.\mathrm{CD}_{3} \mathrm{OD}\right): \delta=39.2,53.6$ (br., $\left.C \mathrm{~B}\right), 62.1,116.0,122.3$, $127.1,129.1,129.6,130.5,131.2,132.1,134.6,139.9,159.6$, $169.6 \mathrm{ppm}, \mathrm{C}-4$ triazole ring not seen. HRMS (ESI-TOF) $\mathrm{m} / \mathrm{z}$ : calcd. for $\mathrm{C}_{18} \mathrm{H}_{19} \mathrm{BN}_{3} \mathrm{O}_{5}[\mathrm{M}+\mathrm{H}]^{+}$368.1416; found 368.1411.

\section{Acknowledgments}

This work was supported by funds provided by the National Institute of Allergy and Infectious Diseases of the National Institutes of Health under award numbers R01 AI100560, and R01 AI063517. The authors also thank Centro Interdipartimentale Grandi Strumenti of Modena (C.I.G.S.) for analytical support.

[1] a) V. M. Dembitsky, A. A. Quntar, M. Srebnik, Mini-Rev. Med. Chem. 2004, 4, 1001-18; b) D. S. Matteson, Med. Res. Rev. 2008, 28, 233-246; c) S. Touchet, F. Carreaux, B. Carboni, A. Bouillon, J. Boucher, Chem. Soc. Rev. 2011, 40, 3895-3914.

[2] a) S. J. Baker, J. W. Tomshob, S. J. Benkovicc, Chem. Soc. Rev. 2011, 40, 4279-4285; b) P. Hunter, EMBO Rep. 2009, 10, 128; c) P. V. Ramachandran, Future Med. Chem. 2013, 5, 611-612.

[3] N. Ni, B. Wang, in: Boronic acids (Ed.: D. G. Hall), 2nd ed., Wiley-VCH, Weinheim, Germany, 2011, p. 591-620.

[4] a) R. Smoum, A. Rubinstein, V. M. Dembitsky, M. Srebnik, Chem. Rev. 2012, 112, 4156-4220; b) B. A. Connolly, D. G. Sanford, A. K. Chiluwal, S. E. Healey, D. E. Peters, M. T. Dimare, W. Wu, Y. Liu, H. Maw, Y. Zhou, Y. Li, Z. Jin, J. L. Sudmeier, J. H. Lai, W. W. Bachovchin, J. Med. Chem. 2008, 51, 6005-6013.

[5] S. Jin, C. Zhu, M. Li, B. Wang, Bioorg. Med. Chem. Lett. 2009, 19, 1596-1599.

[6] a) S. Morandi, F. Morandi, E. Caselli, B.-K. Shoichet, F. Prati, Bioorg. Med. Chem. 2008, 16, 1195-1205; b) O. Eidam, C. Romagnoli, G. Dalmasso, S. Bareliera, E. Caselli, R. Bonnet, B. K. Shoichet, F. Prati, Proc. Natl. Acad. Sci. USA 2012, 109, 17448-17453.

[7] a) H. Li, R. Aneja, I. Chaiken, Molecules 2013, 18, 9797-9817; b) G. C. Tron, T. Pirali, R. A. Billington, P. L. Canonico, G. Sorba, A. A. Genazzani, Med. Res. Rev. 2008, 28, 278-308; c) J. Hou, X. Liu, J. Shen, G. Zhao, P. G. Wang, Expert Opin. Drug Discovery 2012, 7, 489-501; d) A. Lauria, R. Delisi, F. Mingoia, A. Terenzi, A. Martorana, G. Barone, A. M. Almerico, Eur. J. Org. Chem. 2014, 16, 3289-3306.

[8] a) V. V. Rostovtsev, L. G. Green, V. V. Fokin, K. B. Sharpless, Angew. Chem. 2002, 41, 2596-2599; b) M. Meldal, C. W. Tornøe, Chem. Rev. 2008, 108, 2952-3015; c) R. Huisgen, in: 1,3-Dipolar Cycloaddition Chemistry, vol. 1 (Ed.: A. Padwa), Wiley-Interscience, New York, 1984, p. 1-176.

[9] a) H. C. Kolb, K. B. Sharpless, Drug Discovery Today 2003, 8, 1128-1137; b) J. E. Moses, A. D. Moorhouse, Chem. Soc. Rev. 2007, 36, 1249-1262; c) P. Thirumurugan, D. Matosiuk, K. Jozwiak, Chem. Rev. 2013, 113, 4905-4979.

[10] a) M. B. Thathagar, J. Beckers, G. Rothenberg, J. Am. Chem. Soc. 2002, 124, 11858-11859; b) J. Li, J. Li, D. Wang, S. Pi, Y. Xie, M. Zhang, X. Hu, J. Org. Chem. 2007, 72, 2053-2057; c) J. Z. Deng, D. V. Paone, A. T. Ginnetti, H. Kurihara, S. D. Dreher, S. A. Weissman, S. R. Stauffer, C. S. Burgey, Org. Lett. 2009, 11, 345-347; d) D. M. T. Chan, K. L. Monaco, R. Li, D. Bonne, C. G. Clark, P. Y. S. Lam, Tetrahedron Lett. 2003, 44, 3863-3865.

[11] a) S. Jin, G. Choudhary, Y. Cheng, C. Dai, M. Li, B. Wang, Chem. Commun. 2009, 35, 5251-5253; b) C. Dai, Y. Cheng, J. Cui, B. Wang, Molecules 2010, 15, 5768-5781; c) G. A. Molander, J. Ham, Org. Lett. 2006, 8, 2767-2770.

[12] a) J. Huang, S. J. F. Macdonald, A. W. J. Cooper, G. Fisher, J. P. A. Harrity, Tetrahedron Lett. 2009, 50, 5539-5541; b) J. E. 
Grob, J. Nunez, M. A. Dechantsreiter, L. G. Hamann, J. Org. Chem. 2011, 76, 10241-10248.

[13] P. Davoli, R. Fava, S. Morandi, A. Spaggiari, F. Prati, Tetrahedron 2005, 61, 4427-4436.

[14] D. S. Matteson, D. Maliakal, L. Fabry-Asztalos, J. Organomet. Chem. 2008, 693, 2258-2262.

[15] M. Meldal, C. W. Tornøe, Chem. Rev. 2008, 108, 2952-3015.

[16] a) T. Pirali, G. C. Tron, J. Zhu, Org. Lett. 2006, 8, 4145; b)

E. J. Yoo, M. Ahlquist, S. H. Kim, I. Bae, V. V. Fokin, K. B. Sharpless, S. Chang, Angew. Chem. Int. Ed. 2007, 46, 17301733; Angew. Chem. 2007, 119, 1760-1763.
[17] O. Eidam, C. Romagnoli, E. Caselli, K. Babaoglu, D. Teotico Pohlhaus, J. Karpiak, R. Bonnet, B. K. Shoichet, F. Prati, J. Med. Chem. 2010, 53, 7852-7863.

[18] a) D. S. Matteson, R. Ray, R. R. Rocks, D. J. S. Tsai, Organometallics 1983, 2, 1536-1543; b) D. S. Matteson, Acc. Chem. Res. 1988, 21, 294-300.

[19] D. S. Matteson, Chem. Rev. 1989, 89, 1535-1551.

[20] H. E. Gottlieb, V. Kotlyar, A. Nudelman, J. Org. Chem. 1997, 786 $62,7512-7515$.

[21] D. S. Matteson, P. K. Jesthi, K. M. Sadhu, Organometallics 1984, 3, 1284-1288.

Received: October 30, 2014 
[(1,2,3-Triazol-1-yl)methyl]boronic acids, a new potential scaffold for protease inhibitors, have been synthesized by copper-catalyzed azide-alkyne cycloaddition reaction by starting from suitable azidomethylboronates.

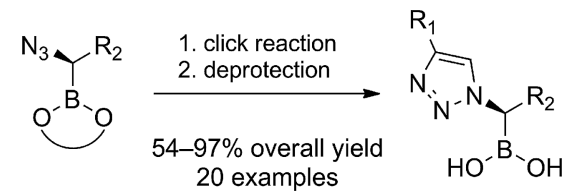

20 examples
C. Romagnoli, E. Caselli,

F. Prati* 1-10

Synthesis of [(1,2,3-Triazol-1-yl)methyl]boronic Acids Through Click Chemistry: Easy Access to a Potential Scaffold for Protease Inhibitors

Keywords: Synthetic methods / Boron / Cycloaddition / Click chemistry / Bioisosters / Medicinal chemistry / Enzyme inhibitors 